\title{
What do we need to probe upper ocean stratification remotely?
}

\author{
Victor I. Shrira and Rema B. Almelah
}

November 4, 2020

\begin{abstract}
We consider whether it is possible in principle to retrieve the key parameters of the mixed layer in the upper ocean (its thickness, bulk eddy viscosity and the pycnocline stratification below) using a theoretical model, which assumes the surface velocity and wind stress to be known from observations. To this end we examine the dynamics of the Ekman current in the novel two-layer model of the upper ocean made of two layers with greatly differing constant eddy viscosities. The presence of stratification manifests itself through suppression of turbulence and, hence, in much smaller value of the eddy viscosity compared to the bulk eddy viscosity $\nu_{e 1}$ in the mixed layer. Within this two-layer model the general solution in terms of explicit Green's function has been derived and analyzed. It was found that a spectral component of frequency $\omega$ of the Ekman current on the surface "feels" the presence of the stratified layer when the mixed layer depth $d$ is less or comparable to the Ekman scale $\sqrt{\frac{2 \nu_{e} 1}{f+\omega}}$, where $f$ is the Coriolis parameter. Thus, under conditions of strong wind resulting in large eddy viscosity $\nu_{e}$, the depth of the mixed layer could be (in principle) inferred from the observations of wind and surface velocity. We conclude by stating, that to retrieve from the wind and surface velocity data the mixed layer parameters, the theoretical model has to be extended by taking into account the effects of the Stokes drift due to surface waves and the possibility of intense mixing at the bottom of the mixed layer.
\end{abstract}

\section{Introduction}

In the upper ocean, the mixed layer and seasonal pycnocline are its most prominent features (e.g. [15], [22]). In the mixed layer between the ocean free surface and the seasonal pycnocline the temperature and salinity is nearly uniform, which led to the term "mixed layer" or "quasiuniform layer". The mixed layer depth is influenced by a variety of physical processes affecting the stratification at its bottom, including, winds, turbulent mixing, radiative heating and cooling. The thickness of the mixed layer varies depending on the external forcing. During the spring and summer there is strong seasonal stratification, the mixed layer is relatively shallow, while it is deeper and less prominent in the autumn and much deeper in winter; the seasonal pycnocline disappears in winter and the mixed layer extends to the main pycnocline. The typical depth 
of the seasonal pycnocline ranges between $20 \mathrm{~m}$ and $200 \mathrm{~m}$. At higher latitudes, the mixed layer depth increases as a result of stronger winds and cooling poleward. The mixed layer is the layer in direct contact with the atmosphere and most active in the air-sea interaction. There is very significant momentum and heat exchange with the atmosphere. Its correct modelling and monitoring its actual state is crucial for weather prediction and climate modelling. The heat content of the mixed layer is one of the key parameters in hurricane modelling ([8]; [18]; [24]). There is an extensive literature on theoretical, numerical and experimental studies on the variety of physical processes in the mixed layer reviewed in $([22])$. However, at present a number of fundamental questions remain open, which makes the accuracy of modelling of the mixed layer dynamics unsatisfactory, especially for many applications concerned with non quasi-geostrophic processes.

The existing measurement techniques (e.g.[22]) mostly represent contact point observations which are too expensive and too spatially sparse. On the other hand, there are rapidly developing remote sensing methods (both satellite e.g. [2], [3] and land based) with excellent spatial coverage. Among the shore-based remote sensing techniques we particularly note the 'High-Frequency (HF) radar' which retrieve surface velocity fields with a high accuracy and good resolution, withe range of up to a few hundred kilometers. Since the EM waves employed in all remote sensing techniques cannot effectively penetrate into the water, we need theoretical models linking the surface currents with the key parameters of the mixed layer. In the literature two ideas were put forward. In ([20]) it was shown that the strong near-inertial oscillations (routinely captured by coastal highfrequency radars) are tightly linked with the presence of shallow density stratification. However, at present, this approach of remote probing of the upper ocean stratification is still very crude, in its current stage it allows one to quantify neither the depth of the pycnocline nor the strength of the stratification, just its presence; it also inconveniently depends on the occurrence of sharp changes of wind needed to generate near-inertial oscillations. The method proposed by Zervakis, Kokkini and Potiris ([28]) is based on the key assumption that the mixed layer responds to wind forcing as a slab-layer. Using remotely retrieved surface velocity provided by a coastal HF radar and concurrent wind-stress data it proved possible to get a good estimate of the mixed layer depth for the specific conditions of the experiment carried out at the Aegean Sea. However, the slab models are known to be very crude and the question - to what extent their predictions could be relied upon outside the very specific environmental conditions of the experiment? - remains outstanding. Thus, from this perspective it is highly desirable to have a better understanding of the links between the wind stress and surface velocity, presumed to be measured remotely with a reasonable accuracy, and the mixed layer depth and the strength of the density stratification below. Of course, it goes without saying, that apart from this particular applied aspect, this outstanding question is of great interest in itself.

Here, we attempt to model the momentum transfer in the upper ocean employing an extension of the Ekman model. In the Ekman type models the turbulence is characterized by a single parameter - eddy viscosity $\nu_{e}$, which can, in principle, be both depth and time dependent. It is well known that density stratification strongly suppresses turbulence (e.g. [17]; [16]), which makes the eddy viscosity in the mixed layer much higher than in the pycnocline (e.g [5]; [26]; [13]). On this basis one layer models with a constant eddy viscosity in the mixed layer and zero viscosity below were put forward (e.g. [12]; [7]). At the bottom of the mixed layer a variety 
of boundary conditions were attempted, a comparison of several possibilities with observations was carried by [7]. Surprisingly, the analysis of the available data did not show a convincing advantage of employing the vanishing shear stress at the bottom of the mixed layer, the most natural boundary condition at the interface, over two other less justified options (no-slip condition or removing the interface alltogether). There might be multiple reasons behind, which we will discuss later. Clearly, there is an open question on what is the right model for the Ekman currents in the mixed layer. Here, as a first step, we adopt the simplest two layer model: two layers with two vastly different constant values of the eddy viscosity. Although such an approach is an obvious oversimplification, it is a significant step forward compared to the slab models.

The second outstanding problem of major interest is how the vertical structure of stratification manifests itself in the surface current under a variable wind. Under what conditions can we expect discernible manifestations? Under what conditions could we employ the stratification surface manifestations for remote sensing of the depth of the mixed layer? It is known that during the passage of hurricanes the mixed layer dramatically deepens, could we explain this phenomenon within the paradigm of the Ekman type-model and link it to the dynamics of surface current? We are not aware of any work addressing these issues.

The specific open questions we aim to clarify are as follows:

(i) Under what conditions and with what accuracy the two-layer Ekman model can be well approximated by a one layer Ekman model with appropriate boundary conditions at the bottom of the mixed layer? What are the appropriate boundary conditions?

(ii) What are the specific effects of stratification on the surface Ekman currents caused by a variable wind? What characteristics of the Ekman current in the near-surface layer depend on the mixed layer thickness? How sensitive is the near surface Ekman current to the viscosity and, hence, to the stratification in the stratified layer?

(iii) Under what conditions there might occur noticeable surface manifestations of the presence of stratification? How do these manifestations depend on the temporal scales under consideration?

(iv) Is it possible to find the depth of the mixed layer having only observations of the surface currents and wind stress? Could it be possible to estimate the strength of the stratification in the pycnocline?

Here, to address the above questions we model the dynamics of the mixed layer caused by a generic time dependent wind forcing focussing upon momentum transfer to the upper ocean within the framework of the Navier-Stokes equations with depth-dependent eddy viscosity. The model is an extension of the classical one-layer Ekman model with a constant eddy viscosity. Here, we consider two layers: the upper one is of depth $d$ with a constant eddy viscosity $\nu_{e 1}$, it is supposed to model the mixed layer; the second layer is characterized by a different constant eddy viscosity $\nu_{e 2}$. We assume the lower layer to be stratified; since stratification suppresses turbulence, the eddy viscosity there is much smaller than in the upper layer, i.e. $\nu_{e 1} \gg \nu_{e 2}$. To focus on the effects due to the presence of stratification, we adopt the simplest model of the 


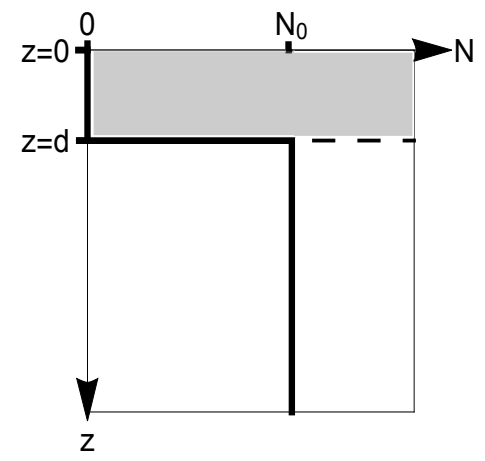

(a)

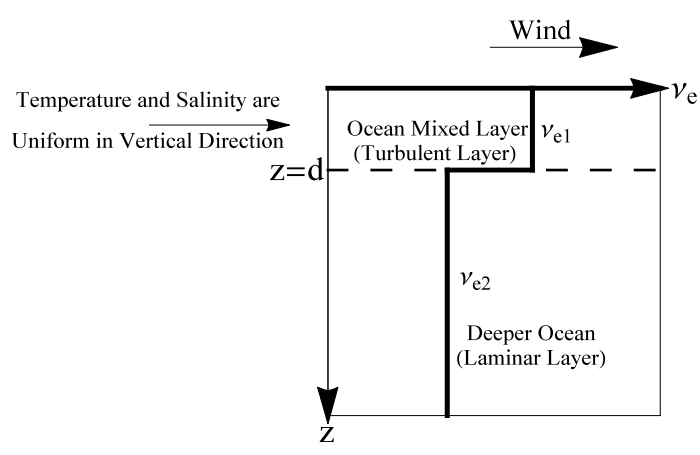

(b)

Figure 1: (a) Sketch of the assumed stratification profile. (b) The model viscosity profile.

mixed layer, assuming the eddy viscosity to be constant both in depth and time. This could be justified, if we understand $\nu_{e 1}$ as a bulk viscosity and do not consider too rapid variations of wind. Since we are primarily interested in the processes in the mixed layer and the transfer of momentum into the ocean interior is very small, we, without much loss of generality, can assume the second layer to be infinitely deep, as sketched in figure (1). We leave aside the issue of specific relationship between the strength of stratification in the second layer characterized by the Brunt-Väisälä frequency $N$ through eddy viscosity $\nu_{2}$. One can use any of the widely used empirical relationships $\nu_{2}(N)$, but to avoid discussion of their merits we just assume the value of $\nu_{e 2}$ to be known and entirely determined by $N$. According to observations, the eddy viscosity in the mixed layer ranges between $O\left(10^{-4}\right)$ and $O\left(10^{-1}\right) \mathrm{m}^{2} \mathrm{~s}^{-1}$ (see table 1 ), while in the pycnocline it is much smaller, varying from $O\left(10^{-6}\right)$ to $O\left(10^{-4}\right) \mathrm{m}^{2} \mathrm{~s}^{-1}$. The table also provides an idea of the variability of the mixed layer thickness.

This paper is organized as follows: $\S 2$ gives the formulation of the mathematical model: a brief description of the equations and boundary conditions governing Ekman currents caused by a time-varying wind. $\S 3$ presents the general solution in terms of Green's function for an

arbitrary time dependence of the wind shear stress. $\S 4$ compares the predictions of the new two-layer model with one-layer solutions derived in [6]; [7] from the remote sensing perspective. Finally, a summary and discussion are provided in the concluding $\S 5$.

\section{The mathematical model}

We begin with the formulation of the Ekman model for two-layer fluid. Here, the fluid is assumed to consist of two layers, as sketched in figure (1): the upper layer of thickness $d$ is adjacent to the surface, it is supposed to model the mixed layer with high eddy viscosity $\nu_{e}$. The second layer with eddy viscosity $\nu_{e 2}$ suppressed by stratification is taken to be infinite, since, as it will be shown below, the horizontal motions caused by varying winds do not penetrate far into the stratified fluid and, therefore, in our context the thickness of the stratified fluid could be assumed infinite without loss of generality. For horizontally uniform motions the corresponding exact reduction of the Navier-Stokes equations (in the Cartesian frame with $z$ directed downward and $z=0$ at 
Table 1: Eddy viscosity values ([9]).

\begin{tabular}{|c|c|c|c|}
\hline Location & Layer depth $m$ & $\nu_{e}, 10^{-4}\left(m^{2} s^{-1}\right)$ & Source \\
\hline \hline Danish Waters & $0-15$ & $1.9-3.8$ & All currents \\
Arctic Ocean & & 160 & Under ice \\
Danish Waters & & $250-1500$ & All currents \\
Kuroshio & $0-200$ & $680-7500$ & All currents \\
Japan Sea & $0-200$ & $150-1460$ & All currents \\
North Siberian Shelf & $0-60$ & $0-1000$ & Tidal currents \\
North Sea & $0-31$ & $75-1720$ & Strong tidal \\
& & & currents \\
Tropical Atlantic & $0-50$ & 320 & Temperature \\
Ocean & & & fluctuation \\
North Siberian Shelf & $0-60$ & $10-400$ & Tidal current \\
Atlantic Ocean 50 $S-10^{\circ} N$ & $0-200$ & $7-50$ & Wind currents \\
Japan Sea & $0-10$ & 100 & Tidal currents \\
Arctic Ocean & $0-100$ & 23.8 & Ice drift \\
North Atlantic & $0-4$ & 146 & Temperature \\
& $4-8$ & 96 & fluctuation \\
Open Ocean & $8-12$ & 47 & \\
Lake Huron & $0-10$ & $150-225$ & Surface \\
Tropical Atlantic & 30 & $65-160$ & Wind current \\
Ocean & $0-12$ & $420 \pm 84$ & Temperature \\
Tropical Atlantic Ocean & $0-10$ & 62 & fluctuation \\
& $10-20$ & 68 & Temperature \\
fluctuation \\
Tropical Atlantic Ocean & $0-12$ & 85 & \\
& $20-50$ & 265 & Temperature \\
fluctuation \\
& & & \\
\hline
\end{tabular}

the unperturbed ocean surface, and $x$ and $y$ directed eastward and northward directions) reads

$$
\begin{aligned}
& \frac{\partial \boldsymbol{U}_{1}}{\partial t}+i \text { f } \boldsymbol{U}_{1}=\nu_{e 1} \frac{\partial^{2} \boldsymbol{U}_{1}}{\partial z^{2}}, \quad 0 \leq z \leq d, \\
& \frac{\partial \boldsymbol{U}_{2}}{\partial t}+i \text { f } \boldsymbol{U}_{2}=\nu_{e 2} \frac{\partial^{2} \boldsymbol{U}_{2}}{\partial z^{2}}, \quad d \leq z<\infty
\end{aligned}
$$

where $\boldsymbol{U}_{1}=u_{1}+i v_{1}$ and $\boldsymbol{U}_{2}=u_{2}+i v_{2}$ are the Reynolds averaged horizontal complex velocities in the mixed layer and the lower stratified layer, respectively, $f=2 \Omega \sin (\phi)$ is the Coriolis 
parameter ( $\Omega$ and $\phi$ are the Earth's rotation frequency and the latitude respectively). As it is standard in the Ekman paradigm, here, to get closed equations for the Reynolds averaged flow we parameterize the Reynolds stresses by adopting the Boussinesq hypothesis, i.e. assume the Reynolds stresses to be proportional to the Reynolds averaged velocity gradient with a single scalar eddy viscosity coefficient, $\nu_{e j}(z, t) \quad(j=1,2)$, constant in each layer.

The motion has to satisfy the continuity of stress boundary conditions at the surface $(z=0)$ and at the interface $(z=d)$, at the interface the velocities have to be continuous as well; the lower boundary condition of decay of the motion at infinity closes the set of boundary conditions:

$$
\begin{array}{rlrl}
\nu_{e} \frac{\partial \boldsymbol{U}_{1}(z, t)}{\partial z} & =\frac{-\boldsymbol{\tau}(t)}{\rho}, & \text { at } z=0, \\
\boldsymbol{U}_{1}(z, t) & =\boldsymbol{U}_{2}(z, t), & \text { at } \quad z=d, \\
\nu_{e 1} \frac{\partial \boldsymbol{U}_{1}(z, t)}{\partial z} & =\nu_{e 2} \frac{\partial \boldsymbol{U}_{2}(z, t)}{\partial z}, & & \text { at } z=d, \\
\boldsymbol{U}_{2}^{\prime}(z, t) & =0, & \text { as } z \rightarrow \infty,
\end{array}
$$

where $\boldsymbol{\tau}(t)$ is the wind stress. The above set of equations provides the mathematical framework of our further analysis.

\section{General solution}

\subsection{Derivation}

In this section we solve the equations of motion (1) by applying the Fourier transform with respect to $t$,

$$
\tilde{\boldsymbol{U}}_{j}(z, \omega)=\int_{-\infty}^{\infty} \boldsymbol{U}_{j}(z, t) e^{-i \omega t} d t ; \quad(j=1,2),
$$

which turns the partial differential equations (1) into ordinary equations with respect to $z$ :

$$
i(f+\omega) \tilde{\boldsymbol{U}}_{1}(z, \omega)-\nu_{e 1} \frac{d^{2} \tilde{\boldsymbol{U}}_{1}(z, \omega)}{d z^{2}}=0, \quad i(f+\omega) \tilde{\boldsymbol{U}}_{2}(z, \omega)-\nu_{e 2} \frac{d^{2} \tilde{\boldsymbol{U}}_{2}(z, \omega)}{d z^{2}}=0 .
$$

By using the Fourier transformed boundary conditions:

$$
\begin{aligned}
\nu_{e 1} \frac{\partial \tilde{\boldsymbol{U}}_{1}(z, \omega)}{\partial z} & =\frac{-\boldsymbol{\tau}(\omega)}{\rho}, & \text { at } z=0 \\
\tilde{\boldsymbol{U}}_{1}(z, \omega) & =\tilde{\boldsymbol{U}}_{2}(z, \omega), & \text { at } z=d \\
\nu_{e 1} \frac{\partial \tilde{\boldsymbol{U}}_{1}(z, \omega)}{\partial z} & =\nu_{e 2} \frac{\partial \tilde{\boldsymbol{U}}_{2}(z, \omega)}{\partial z}, & \text { at } z=d \\
\tilde{\boldsymbol{U}}_{2}^{\prime}(z, \omega) & =0, & \text { as } z \rightarrow \infty
\end{aligned}
$$


the solutions for $\tilde{\boldsymbol{U}}_{1}(z, \omega)$ and $\tilde{\boldsymbol{U}}_{2}(z, \omega)$ are sought in the form:

$$
\tilde{\boldsymbol{U}}_{1}(z, \omega)=\boldsymbol{\tau}(\omega) \boldsymbol{K}_{1}(z, \omega), \quad \tilde{\boldsymbol{U}}_{2}(z, \omega)=\boldsymbol{\tau}(\omega) \boldsymbol{K}_{2}(z, \omega)
$$

Then the general solution of equations (1) as function of $z, t$ is given by the inverse Fourier transform,

$$
\boldsymbol{U}_{j}(z, t)=\frac{1}{2 \pi} \int_{-\infty}^{\infty} \tilde{\boldsymbol{U}}_{j}(z, \omega) e^{i \omega t} d \omega ; \quad j=1,2
$$

The general solution to the equations of motion in the Fourier space (4) can be written as,

$$
\begin{aligned}
& \tilde{\boldsymbol{U}}_{1}(z, \omega)=A \exp \left[(1+i) z / \delta_{1}(\omega)\right]+B \exp \left[-(1+i) z / \delta_{1}(\omega)\right] \\
& \tilde{\boldsymbol{U}}_{2}(z, \omega)=c_{1} \exp \left[(1+i) z / \delta_{2}(\omega)\right]+c_{2} \exp \left[-(1+i) z / \delta_{2}(\omega)\right]
\end{aligned}
$$

where,

$$
\delta_{1}(\omega)=\sqrt{\frac{2 \nu_{e 1}}{f+\omega}} \text { and } \delta_{2}(\omega)=\sqrt{\frac{2 \nu_{e 2}}{f+\omega}} .
$$

The quantities $\delta_{1}(\omega)$ and $\delta_{2}(\omega)$ are the frequency dependent Ekman scales for the first and the second layer, they determine how deep into the layer a perturbation of frequency $\omega$ penetrates. Usually, $\delta_{2}(\omega) \ll \delta_{1}(\omega)$ and $\delta_{2}(\omega)$ is so small, that the assumption that the stratified layer is infinite, is justified.

By applying the transformed boundary conditions at the surface and at infinity, the general solution for each layer in the Fourier space can be rewritten as,

$$
\tilde{\boldsymbol{U}}_{1}=2 B \cosh \left[(1+i) z / \delta_{1}(\omega)\right]+\frac{\boldsymbol{\tau}(\omega) e^{-i \pi / 4}}{\rho \sqrt{\nu_{1}} \sqrt{f+\omega}} \exp \left[-(1+i) z / \delta_{1}(\omega)\right]
$$

and,

$$
\tilde{\boldsymbol{U}}_{2}(z, \omega)=c_{2} \exp \left[-(1+i) z / \delta_{2}(\omega)\right] .
$$

The unspecified yet arbitrary constants $B$ and $c_{2}$ are determined from the boundary conditions at the internal interface:

$$
\begin{aligned}
& B=\frac{\left(\sqrt{\nu_{e 1}}+\sqrt{\nu_{e 2}}\right) \boldsymbol{\tau}(\omega) e^{-i \pi / 4} \exp \left[(1+i) d / \delta_{1}(\omega)\right]}{2 \rho \sqrt{\nu_{e 1}} \sqrt{f+\omega}\left(\sqrt{\nu_{e 2}} \cosh \left[(1+i) d / \delta_{1}(\omega)\right]+\sqrt{\nu_{e 1}} \sinh \left[(1+i) d / \delta_{1}(\omega)\right]\right)}, \\
& c_{2}=\frac{\boldsymbol{\tau}(\omega) e^{-i \pi / 4} \exp \left[(1+i) d / \delta_{2}(\omega)\right]}{\rho \sqrt{f+\omega}\left(\sqrt{\nu_{e 2}} \cosh \left[(1+i) d / \delta_{1}(\omega)\right]+\sqrt{\nu_{e 1}} \sinh \left[(1+i) d / \delta_{1}(\omega)\right]\right)} .
\end{aligned}
$$

Finally, the general solution in the upper and lower layers is as follows: 


$$
\boldsymbol{U}_{1}(z, t)=\frac{1}{2 \pi} \int_{-\infty}^{\infty} \tilde{\boldsymbol{U}}_{1}(z, \omega) e^{i \omega t} d \omega, \quad \boldsymbol{U}_{2}(z, t)=\frac{1}{2 \pi} \int_{-\infty}^{\infty} \tilde{\boldsymbol{U}}_{2}(z, \omega) e^{i \omega t} d \omega
$$

where

$$
\tilde{\boldsymbol{U}}_{1}(z, \omega)=\frac{e^{-i \pi / 4} \boldsymbol{\tau}(\omega)\left(\sqrt{\nu_{e 1}} \cosh \left[(1+i)(d-z) / \delta_{1}(\omega)\right]+\sqrt{\nu_{e 2}} \sinh \left[(1+i)(d-z) / \delta_{1}(\omega)\right]\right)}{\rho \sqrt{\nu_{e 1}} \sqrt{f+\omega}\left(\sqrt{\nu_{e 2}} \cosh \left[(1+i) d / \delta_{1}(\omega)\right]+\sqrt{\nu_{e 1}} \sinh \left[(1+i) d / \delta_{1}(\omega)\right]\right)}
$$

and,

$$
\tilde{\boldsymbol{U}}_{2}(z, \omega)=\frac{e^{-i \pi / 4} \boldsymbol{\tau}(\omega) \exp \left[(1+i)(d-z) / \delta_{2}(\omega)\right]}{\rho \sqrt{f+\omega}\left(\sqrt{\nu_{e 2}} \cosh \left[(1+i) d / \delta_{1}(\omega)\right]+\sqrt{\nu_{e 1}} \sinh \left[(1+i) d / \delta_{1}(\omega)\right]\right)} .
$$

In the limit $\nu_{2} \rightarrow 0$ the solution above tends to the one-layer solution with the boundary condition $\boldsymbol{U}^{\prime}=0$ at the bottom of the mixed layer derived in $([7])$. Below we analyse important particular cases and some implications of the solution derived above.

\subsection{Particular regimes, the fast $(\omega \gg f)$ and slow $(\omega \ll f)$ timescales}

So far we have not used any explicit assumptions regarding smallness of $\nu_{2}$ compared to $\nu_{e 1}$ (although we made an implicit assumption by setting the thickness of the stratified layer to be infinite). Here we consider $\nu_{e 2} / \nu_{e 1} \ll 1$ and, in particular, examine behaviour of the Ekman response in the limit $\nu_{e 2} / \nu_{e 1} \rightarrow 0$. In this section we also examine particular regimes where the thickness of the mixed layer is either small or large compared to the frequency dependent Ekman scale.

\section{General case}

For $\nu_{e 1} \gg \nu_{e 2}$, the constants $B$ and $c_{2}$ in equations (11) simplify to:

$$
B=\frac{\boldsymbol{\tau}(\omega) e^{-i \pi / 4} \exp \left[(1+i) d / \delta_{1}(\omega)\right]}{2 \rho \sqrt{f+\omega} \sqrt{\nu_{e 1}} \sinh \left[(1+i) d / \delta_{1}(\omega)\right]}, \quad c_{2}=\frac{\boldsymbol{\tau}(\omega) e^{-i \pi / 4} \exp \left[(1+i) d / \delta_{2}(\omega)\right]}{\rho \sqrt{f+\omega} \sqrt{\nu_{e 1}} \sinh \left[(1+i) d / \delta_{1}(\omega)\right]},
$$

so that, the general solution in the upper and lower layer in the Fourier space takes the form:

$$
\tilde{\boldsymbol{U}}_{1}(z, \omega)=\frac{\boldsymbol{\tau}(\omega) e^{-i \pi / 4} \cosh \left[(1+i)(d-z) / \delta_{1}(\omega)\right]}{\rho \sqrt{\nu_{e 1}} \sqrt{f+\omega} \sinh \left[(1+i) d / \delta_{1}(\omega)\right]} .
$$

and,

$$
\tilde{\boldsymbol{U}}_{2}(z, \omega)=\frac{\boldsymbol{\tau}(\omega) e^{-i \pi / 4} \exp \left[(1+i)(d-z) / \delta_{2}(\omega)\right]}{\rho \sqrt{\nu_{e 1}} \sqrt{f+\omega} \sinh \left[(1+i) d / \delta_{1}(\omega)\right]} .
$$

The resulting transfer function in the upper layer is the same as in the one-layer Elipot and Gille model ([7]) with velocity shear vanishing at $z=d([7])$. Note, that the solution of the Elipot and Gille model [7] is obtained under the assumption of the total suppression of turbulence in the stratified layer and, hence, vanishing of the eddy viscosity there, was also derived in an explicit form for the case of a sharp increase of wind in [12]. 
Shallow mixed layer $\left(z, d \ll \delta_{1}(\omega)\right)$

The solution in the upper layer, $\tilde{\boldsymbol{U}}_{1}$, with arbitrary $\nu_{e 1}$ and $\nu_{e 2}$ is:

$$
\begin{aligned}
\tilde{\boldsymbol{U}}_{1}(z, \omega) & =\frac{\boldsymbol{\tau}(\omega) e^{-i \pi / 4}\left(\sqrt{\nu_{e 1}}+\sqrt{\nu_{e 2} / \nu_{e 1}} e^{i \pi / 4}(d-z) \sqrt{f+\omega}\right)}{\rho \sqrt{\nu_{e 1}} \sqrt{f+\omega}\left(\sqrt{\nu_{e 2}}+e^{i \pi / 4} d \sqrt{f+\omega}\right)} \\
& =\frac{\boldsymbol{\tau}(\omega) e^{-i \pi / 4}\left(\sqrt{\nu_{e 1}}+(1+i) \sqrt{\nu_{e 2}}(d-z) / \delta_{1}(\omega)\right)}{\rho \sqrt{\nu_{e 1}} \sqrt{f+\omega}\left(\sqrt{\nu_{e 2}}+e^{i \pi / 4} d \sqrt{f+\omega}\right)} .
\end{aligned}
$$

For $\nu_{e 1} \gg \nu_{e 2}$ the expression for $\tilde{\boldsymbol{U}}_{1}(z, \omega)$ can be simplified to,

$$
\tilde{\boldsymbol{U}}_{1}(z, \omega)=\frac{\boldsymbol{\tau}(\omega) e^{-i \pi / 4}}{\rho \sqrt{f+\omega}\left(\sqrt{\nu_{e 2}}+e^{i \pi / 4} d \sqrt{f+\omega}\right)} .
$$

For the shallow mixed layer, $d / \delta_{1} \ll 1$, the flow in the second layer does not feel the presence of the mixed layer and we recover the Ekman solution for the one layer ocean with viscosity $\nu_{e 2}$ :

$$
\tilde{\boldsymbol{U}}_{2}=\frac{\boldsymbol{\tau}(\omega) e^{-i \pi / 4}}{\rho \sqrt{\nu_{e 2}(f+\omega)}} \exp \left[-(1+i) z \sqrt{\frac{f+\omega}{2 \nu_{e 2}}}\right], \quad\left(\left.\tilde{U}_{2}\right|_{z=0}=2 B, \quad B=\frac{\boldsymbol{\tau}(\omega) e^{-i \pi / 4}}{2 \rho \sqrt{\nu_{e 2}(f+\omega)}}\right) .
$$

For the slow time scales $(\omega \ll f)$ the solution can be simplified even further,

$$
\tilde{\boldsymbol{U}}_{1}=\frac{\boldsymbol{\tau}(\omega) e^{-i \pi / 4}}{\rho \sqrt{\nu_{e 2} f}}, \quad \tilde{\boldsymbol{U}}_{2}=\frac{\boldsymbol{\tau}(\omega) e^{-i \pi / 4}}{\rho \sqrt{\nu_{e 2} f}} \exp \left[-(1+i) z \sqrt{\frac{f}{2 \nu_{e 1}}}\right] .
$$

\section{Deep mixed layer $\left(d \gg \delta_{1}\right)$}

The transfer function for the upper layer takes the form,

$$
\tilde{\boldsymbol{U}}_{1}(z, \omega)=\frac{\boldsymbol{\tau}(\omega) e^{-i \pi / 4}}{\rho \sqrt{\nu_{e 1}(f+\omega)}} \exp \left[-(1+i) z \sqrt{\frac{f+\omega}{2 \nu_{e 1}}}\right], \quad\left(B=\frac{\boldsymbol{\tau}(\omega) e^{-i \pi / 4}}{\rho \sqrt{\nu_{e 1}(f+\omega)}}\right),
$$

which coincides with the transfer function of the classical one-layer Ekman model. For the fast processes $(\omega \gg f)$ the effect of rotation is negligible and the flow can be considered unidirectional. The transfer function for the lower layer is expectedly vanishingly small,

$$
\tilde{\boldsymbol{U}}_{2}(z, \omega)=\frac{\boldsymbol{\tau}(\omega) e^{-i \pi / 4}}{\rho \sqrt{\nu_{e 1}(f+\omega)}} \frac{\exp \left[(1+i)(d-z) / \delta_{2}(\omega)\right]}{\exp \left[(1+i) d / \delta_{1}(\omega)\right]} .
$$




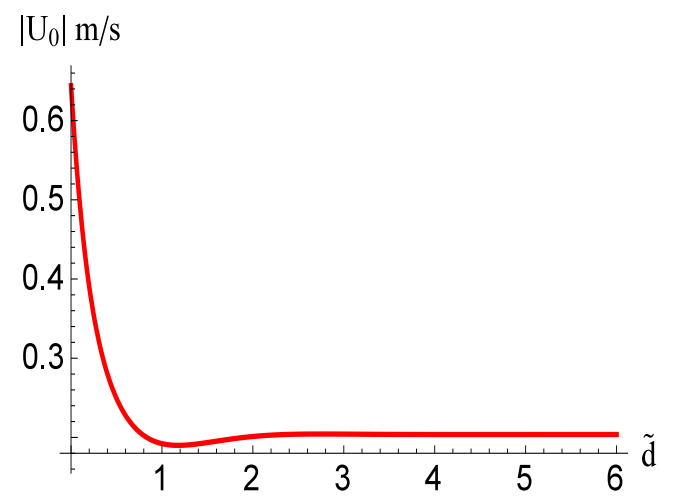

(a)

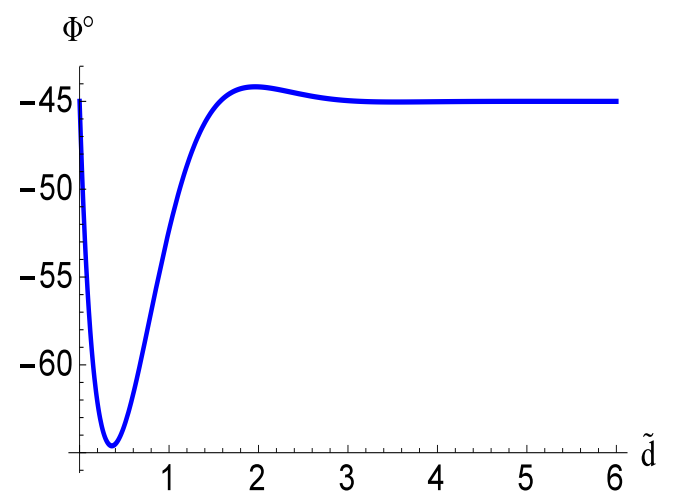

(b)

Figure 2: Dependence of the steady Ekman current at the ocean surface $\boldsymbol{U}_{0}$ on the mixed layer non-dimensional depth $\tilde{d}=d / \delta_{1}$ : (a) Surface current speed. (b) Deflection of the current direction at the surface with respect to wind. The sample parameters are $\nu_{e 1}=7 \times 10^{-3} \mathrm{~m}^{2} \mathrm{~s}^{-1}$ (which corresponds to $10 \mathrm{~m} \mathrm{~s}^{-1}$ wind) , $\nu_{e 2}=7 \times 10^{-4} \mathrm{~m}^{2} \mathrm{~s}^{-1}, f=10^{-4} \mathrm{~s}^{-1}, \rho=1027 \mathrm{~kg} \mathrm{~m}^{-3}$ and $\tau_{0}=0.175 \mathrm{~N} \mathrm{~m}^{-2}$.

\subsection{Steady-state solution}

It is helpful to examine in more details the steady solutions, as the simplest case of the general

solution discussed above. Under a constant wind $\left(\boldsymbol{\tau}=\boldsymbol{\tau}_{0}\right)$, the general solution describing the Ekman current simplifies to become,

$$
\begin{aligned}
& \boldsymbol{U}_{1}(z)=\frac{\boldsymbol{\tau}_{0} e^{-i \pi / 4}\left(\sqrt{\nu_{e 1}} \cosh \left[(1+i)(d-z) / \delta_{1}(0)\right]+\sqrt{\nu_{e 2}} \sinh \left[(1+i)(d-z) / \delta_{1}(0)\right]\right)}{\rho \sqrt{\nu_{e 1} f}\left(\sqrt{\nu_{e 2}} \cosh \left[(1+i) d / \delta_{1}(0)\right]+\sqrt{\nu_{e 1}} \sinh \left[(1+i) d / \delta_{1}(0)\right]\right)}, \\
& \boldsymbol{U}_{2}(z)=\frac{\boldsymbol{\tau}_{0} e^{-i \pi / 4} \exp \left[(1+i)(d-z) / \delta_{2}(0)\right]}{\rho \sqrt{f}\left(\sqrt{\nu_{e 2}} \cosh \left[(1+i) d / \delta_{1}(0)\right]+\sqrt{\nu_{e 1}} \sinh \left[(1+i) d / \delta_{1}(0)\right]\right)} .
\end{aligned}
$$

When $d \ll \delta_{1}(0)$, equation for the Ekman current at the surface $z=0$ (surface current velocity, $\boldsymbol{U}_{1}$, and the angle $\Phi$ of the current deflection relative to the wind) becomes:

$$
\boldsymbol{U}_{1}(0)=\frac{\boldsymbol{\tau}_{0} e^{-i \pi / 2}}{\rho f d}\left(1-\frac{e^{-i \pi / 4} \sqrt{\nu_{e 2}}}{d \sqrt{f}}\right), \quad \Phi=\tan ^{-1}\left(d \sqrt{\frac{2 f}{\nu_{e 2}}}-1\right) .
$$

Figure 2 illustrates the sensitivity of the steady Ekman current at the surface to the nondimensional depth of the mixed layer $\tilde{d}=d / \delta_{1}$. Since $\delta_{1}$ scales with wind as $U_{10}^{2}$ or, equivalently, as $u_{*}^{2}$, the surface currents feels the stratification only under strong winds, when, roughly, $d / \delta_{1} \leq 1$. Typical samples of velocity components $(u, v)$ and are shown in figure 3 . The solution (23) and figure 3 most clearly illustrate the point that the Ekman currents do not behave as it is assumed in the slab models, it is less straightforward to see this in the general solution. Thus, when we are interested in ageostrpophic Ekman motions the use of the slab models is 
problematic.

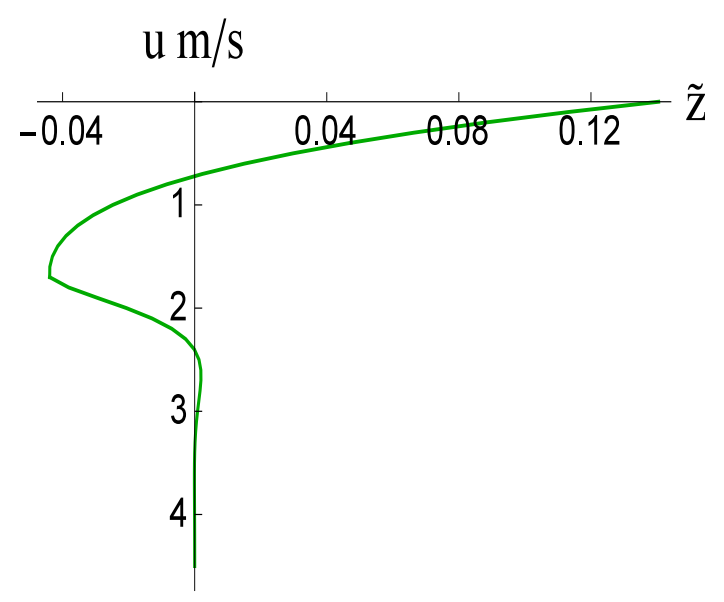

(a)

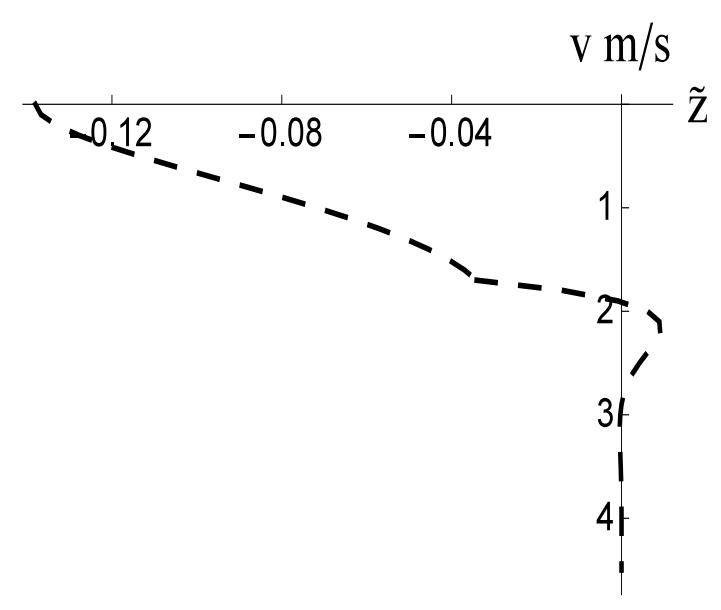

(b)

Figure 3: (a,b) The $x$ and $y$ velocity components vs $\tilde{z}=z / \delta_{1}$ for $\tilde{d}=1.7$. The parameter values are: $\nu_{e 1}=7 \times 10^{-3} \mathrm{~m}^{2} \mathrm{~s}^{-1}, \nu_{e 2}=7 \times 10^{-4} \mathrm{~m}^{2} \mathrm{~s}^{-1}, f=10^{-4} \mathrm{~s}^{-1}, \rho=1027 \mathrm{~kg} \mathrm{~m}^{-3}$ and $\tau_{0}=0.175 \mathrm{~N} \mathrm{~m}^{-2}$.

\subsection{Comparison with the one-layer models}

The solution found and analyzed in the previous section provides full description of the Ekman currents caused by a given time dependent wind within the framework of the two-layer model. To understand how the surface currents depend on the parameters of the upper ocean and whether it might be feasible to find these parameters we have to compare the predictions of our two-layer model with those of the available much simpler one layer models. In this section we compare the predictions of our two-layer model and the established one-layer models: (a) The Elipot \& Gille model ([7]) which assumes infinitely strong stratification $\left(\nu_{e 2}=0\right)$, (b) the classical Ekman model with no stratification, constant viscosity and infinite depth of the ocean.

Figures 4 and 5 illustrate sensitivity of vertical profiles of velocity to the strength of stratification characterized by $\nu_{e 2}$ and to the eddy viscosity in the mixed layer $\nu_{e 1}$. The figures are plotted for the nondimensional depth of the mixed layer $\tilde{d}=d / \delta_{1}=1$. The upper/lower panels illustrate the sensitivity of velocity profiles to the stratification for two sample values of the viscosity in the mixed layer $\nu_{e 1}$ equal to $\nu_{e 1}=5 \times 10^{-3} \mathrm{~m}^{2} \mathrm{~s}^{-1}$ and $\nu_{e 1}=7 \times 10^{-3} \mathrm{~m}^{2} \mathrm{~s}^{-1}$. Note that $\tilde{z}$ is the normalized depth $\tilde{z}=z / \delta_{1}$.

As expected, the predictions of the two-layer model are in between the predictions of the Elipot \& Gille model ([7]) corresponding to infinitely strong stratification in the second layer and the classical Ekman model ([6]) which corresponds to infinite depth of the mixed layer. We could see that, overall, the dependence on $\nu_{e 2}$ is relatively weak (see figure 4), but the effect of 


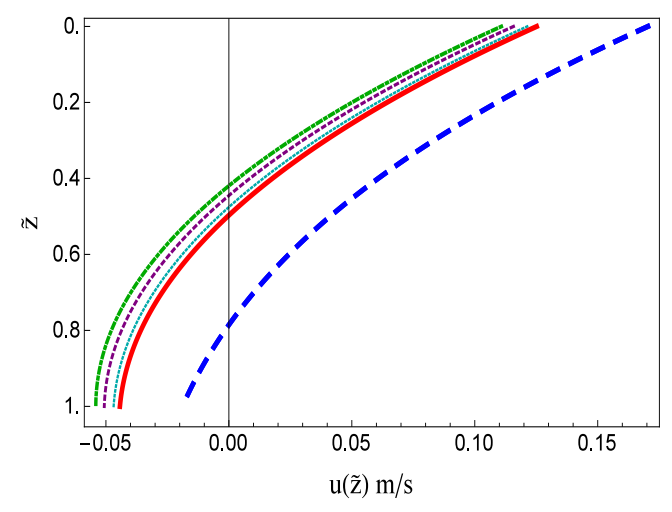

$-v_{\mathrm{e} 2}=10^{-4} \ldots \ldots \ldots v_{\mathrm{e} 2}=5 \times 10^{-5} \ldots v_{\mathrm{e} 2}=10^{-5}$

(a)

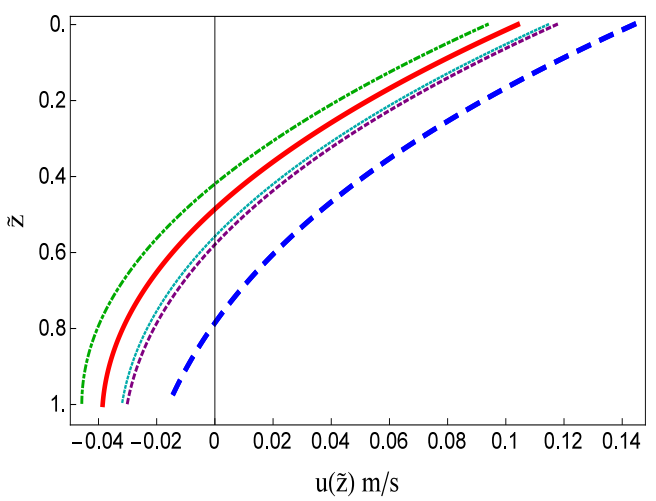

$-v_{\mathrm{e} 2}=10^{-4} \ldots \ldots \ldots v_{\mathrm{e} 2}=5 \times 10^{-4} \ldots v_{\mathrm{e} 2}=7 \times 10^{-4}$

(c)

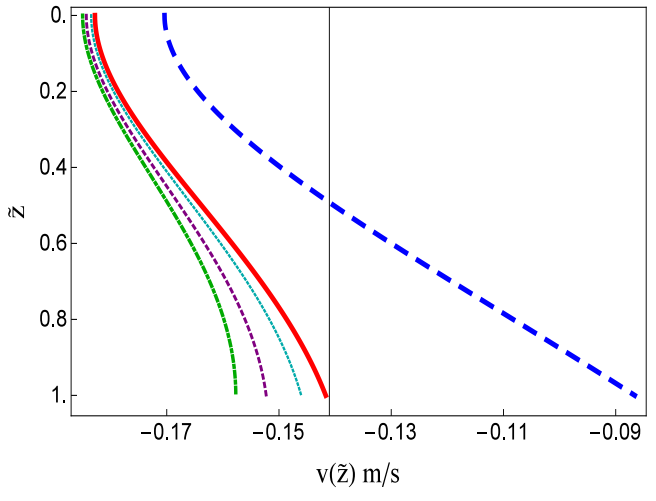

$-v_{\mathrm{e} 2}=10^{-4} \quad \ldots \ldots . . v_{\mathrm{e} 2}=5 \times 10^{-5} \quad \ldots \ldots v_{\mathrm{e} 2}=10^{-5}$

(b)

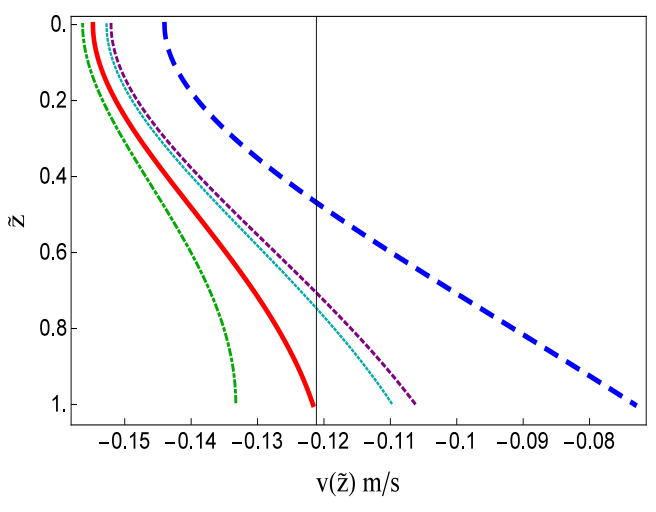

$-v_{\mathrm{e} 2}=10^{-4} \ldots \ldots \ldots v_{\mathrm{e} 2}=5 \times 10^{-4} \ldots v_{\mathrm{e} 2}=7 \times 10^{-4}$

(d)

Figure 4: Sensitivity of vertical profiles of velocity components $u(\tilde{z}), v(\tilde{z})$ to the strength of stratification characterized by $\nu_{e 2}$. Comparison between the steady-state solutions for the classical Ekman model( blue dashed line) and the Elipot and Gille model (green dot-dashed line). $(\mathrm{a}, \mathrm{b}): \nu_{e 1}=5 \times 10^{-3} \mathrm{~m}^{2} \mathrm{~s}^{-1} .(\mathrm{c}, \mathrm{d}): \nu_{e 1}=7 \times 10^{-3} \mathrm{~m}^{2} \mathrm{~s}^{-1} \cdot \tilde{d}=1, f=10^{-4} \mathrm{~s}^{-1}, \rho=1027 \mathrm{~kg} \mathrm{~m}{ }^{-3}$ and $\tau_{0}=0.175 \mathrm{~N} \mathrm{~m}^{-2}$. 


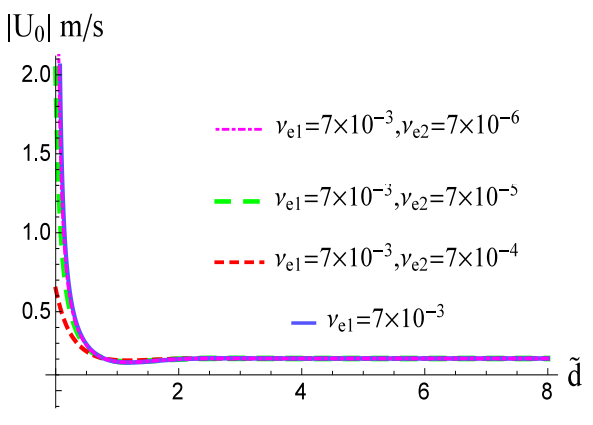

(a)

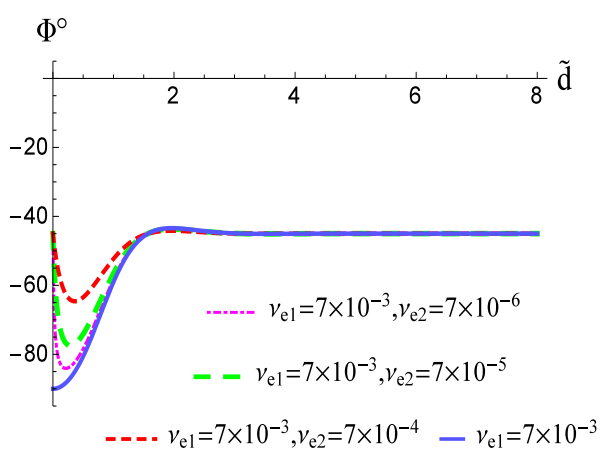

(b)

Figure 5: Sensitivity of the dependence of the steady Ekman current at the surface on the mixed layer thickness and the stratification below. Comparison between the steady-state solutions for Elipot and Gille model (solid line) and two-layer model (dashed and dot-dashed lines): (a),(b) Dependence of the Ekman current speed and direction at the surface on nondimensional mixed layer depth $\tilde{d}$ for the two-layer model with a sample set of parameters : $f=10^{-4} \mathrm{~s}^{-1}, \rho=$ $1027 \mathrm{~kg} \mathrm{~m}^{-3}$ and $\tau_{0}=0.175 \mathrm{~N} \mathrm{~m}^{-2}$.

the presence of stratification is quite robust for a wide range of stratifications. Although plots in figure (4) are made for the single value of the nondimensional depth of the mixed layer, $\tilde{d}=1$, these conclusions hold for all $\tilde{d}=O(1)$. Figure (5) illustrates the surface velocity dependence on the nondimensional depth of the mixed layer, $\tilde{d}$. It shows that the presence of stratification can significantly affect the surface velocity magnitude and direction, only when, roughly, $\tilde{d} \lesssim 1$. Moreover, for such shallow mixed layers the dependence on the strength of the stratification is robust and, can, be potentially, used for retrieving the stratification. The condition on the thickness of the mixed layer $(\tilde{d} \lesssim 1)$ can be satisfied either for a shallow diurnal-type thermocline occurring under light wind and strong solar heating in the first few meters of the water column (e.g. [20], [10]), or under conditions of very strong wind characterized by large values of $\nu_{e 1}$.

\section{Comparison of predictions of three models: the two-layer, Ekman (1905) and Elipot \& Gille (2009)}

Here we further elaborate the discrepancies between the predictions of the Elipot and Gille solution with the vanishing stress at the interface and the classical Ekman solution, we focus upon discrepancies at the surface. We normalize these discrepancies by the Ekman solution at the surface. First we recall these solutions. We begin with the steady solutions. The classical Ekman solution $\left(\boldsymbol{U}_{C E}\right)$ and the Elipot and Gille solution $\left(\boldsymbol{U}_{E G}\right)$ with the vanishing stress at the interface are, respectively,

$$
\boldsymbol{U}_{C E}(z)=\frac{\boldsymbol{\tau}_{0} e^{-i \pi / 4}}{\rho \sqrt{\nu_{e 1} f}} \exp [-(1+i) \tilde{z}]
$$




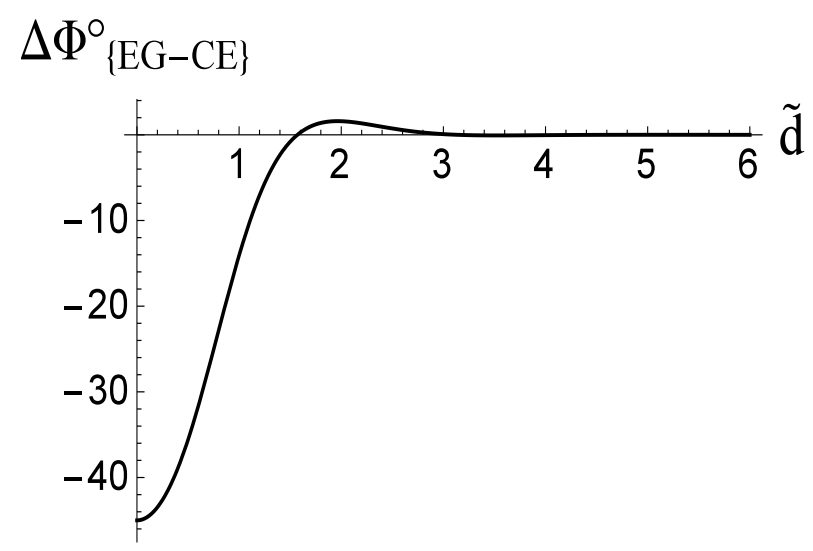

Figure 6: The discrepancy in the surface velocity deflection angle between the Elipot and Gille solution and the classical Ekman solution for a steady wind $\left(\Delta \Phi_{\{E G-C E\}}=\Phi_{E G}+\pi / 4\right)$. $\Phi_{E G}$ is given by eq.(28).

and,

$$
\boldsymbol{U}_{E G}(z)=\frac{\boldsymbol{\tau}_{0} e^{-i \pi / 4}}{\rho \sqrt{\nu_{e 1} f}} \frac{\cosh [(1+i)(\tilde{d}-\tilde{z})]}{\sinh [(1+i) \tilde{d}]}, \quad\left(\tilde{z}=z / \delta_{1}(0) \quad \text { and } \quad \tilde{d}=d / \delta_{1}(0)\right) .
$$

At at the surface, $z=0$, the Elipot and Gille solution (26) yields,

$$
\boldsymbol{U}_{E G}(0)=\frac{\boldsymbol{\tau}_{0} e^{-i \pi / 4}}{\rho \sqrt{f \nu_{e 1}}} \operatorname{coth}[(1+i) \tilde{d}]=\frac{\boldsymbol{\tau}_{0} e^{-i \pi / 4}}{\rho \sqrt{f \nu_{e 1}}}\left(\frac{i \sin (2 \tilde{d})-\sinh (2 \tilde{d})}{\cos (2 \tilde{d})-\cosh (2 \tilde{d})}\right) .
$$

The explicit expressions for the surface current speed and the surface velocity deflection angle with respect to wind take the form,

$$
\left|\boldsymbol{U}_{E G}(\tilde{d})\right|=\frac{\boldsymbol{\tau}_{0}}{\rho \sqrt{f \nu_{e 1}}}\left(\frac{\cosh (2 \tilde{d})+\cos (2 \tilde{d})}{\cosh (2 \tilde{d})-\cos (2 \tilde{d})}\right)^{1 / 2}, \quad \tan \left(\Phi_{E G}(\tilde{d})\right)=\frac{\sin (2 \tilde{d})+\sinh (2 \tilde{d})}{\sin (2 \tilde{d})-\sinh (2 \tilde{d})}
$$

The discrepancy, $\Delta \Phi_{\{E G-C E\}}^{\circ}$, between the velocity deflection angle given by equation (28) and the deflection angle at the surface for the classical Ekman model is a measure of the maximal possible effect of stratification, a rough estimate. The dependence of this discrepancy on the nondimensional mixed layer depth $\tilde{d}$ is shown in figure 6 .

To quantify the comparison between the predictions of the Elipot and Gille model and the classical Ekman model we introduce a relative discrepancy $\delta_{\{E G-C E\}}$ :

$$
\delta_{\{E G-C E\}}=\left|\boldsymbol{\Delta}_{\{E G-C E\}}\right|=\frac{\boldsymbol{U}_{E G}(0)-\boldsymbol{U}_{C E}(0)}{\left|\boldsymbol{U}_{C E}(0)\right|} .
$$

On introducing notation $\zeta=(1+i) \tilde{d}$ and assuming $\left|e^{-\zeta}\right| \ll 1$ we can get a simple rough estimate for the modulus of this discrepancy. To leading order in $\left|e^{-\zeta}\right|$,

$$
\delta_{\{E G-C E\}} \approx 2\left|e^{-2 \zeta}\right|
$$




\begin{tabular}{|c|c|c|c|c|}
\hline$U_{10}\left(\mathrm{~m} \mathrm{~s}^{-1}\right)$ & $\tau\left(\mathrm{N} \mathrm{m}^{-2}\right)$ & $u_{\star}\left(\mathrm{m} \mathrm{s}^{-1}\right)$ & $\nu_{e} \mathrm{~m}^{2} \mathrm{~s}^{-1}$ & $\delta_{1}(\mathrm{~m})$ \\
\hline 5 & 0.044 & 0.007 & 0.01 & 16 \\
10 & 0.175 & 0.013 & 0.05 & 30 \\
30 & 1.575 & 0.039 & 0.5 & 100 \\
50 & 4.375 & 0.065 & 1.3 & 160 \\
\hline
\end{tabular}

Table 2: Estimated eddy viscosity coefficients and the depth scale of the Ekman layer where $\delta_{1}=\sqrt{2 \nu_{e 1} / f}, \quad \nu_{e 1}=c u_{\star}^{2} / f, \quad c=0.03, \quad u_{\star}=\sqrt{\tau / \rho_{w}}, \quad \tau=\rho_{a} U_{10}^{2} C_{D}, \quad \rho_{a}=1.25 \mathrm{~kg} \mathrm{~m}^{-3}$, $C_{D}=1.4 \times 10^{-3}, \rho_{w}=1027 \mathrm{~kg} \mathrm{~m}^{-3}$, and $f=10^{-4} \mathrm{~s}^{-1}([4])$.

Let us introduce the 'critical thickness' $\tilde{d}_{\delta}$ as the threshold thickness of the mixed layer at which a strong stratification localized just below would produce in the surface velocity field an a priori chosen discrepancy $\delta$ with the predictions of the classical Ekman model,

$$
\tilde{d}_{\delta}=\frac{1}{2} \ln \left(\frac{2}{\delta_{\{E G-C E\}}}\right) .
$$

If, for example, we set the threshold value $\delta_{\{E G-C E\}}$ to be 0.1 , then, according to our rough estimate (30), the surface current will be sensitive to stratification when $\tilde{d} \leq 1.5$. Since $\tilde{d}$ depends on $\nu_{1}$, which scales as $u_{*}^{2}$ or $U_{10}^{2}$, the critical depth of the mixed layer at which the current at the surface begins to feel the stratification increases linearly with wind increase. Depending on the chosen threshold value of the discrepancy $\delta_{\{E G-C E\}}$ and on the weather conditions determining $\nu_{e 1}$ which we presume to be given, we can estimate the critical value of the mixed layer thickness $\tilde{d}_{\delta}$ at which the chosen value of $\delta_{\{E G-C E\}}$ occurs. That is, figures 6,5 suggest that depending on $\delta_{\{E G-C E\}}$ we choose, we could see manifestations of the stratification in perturbation of the surface velocity at values of $\tilde{d}$ not exceeding 1-1.5. Recall, that $\tilde{d}=d / \delta_{1}$ and $\delta_{1}$ scales as $u_{*}^{2}$. Table (2) gives an idea on the eddy viscosity and $\delta_{1}$ for winds varying from a gentle breeze to hurricane.

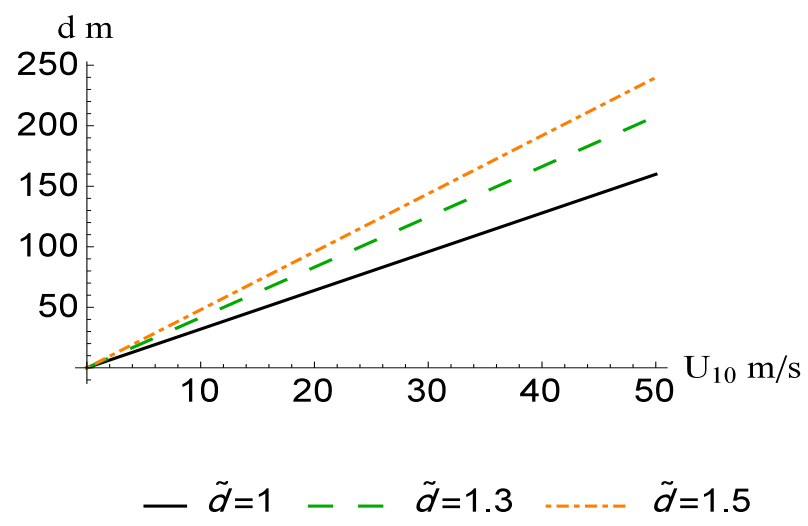

Figure 7: The depth of the mixed layer for typical critical values of $\tilde{d}=d / \delta_{1}$ 
It is also helpful to provide an explicit estimate of the critical $d$ in meters for the whole range of possible wind strengths. Figure 7 sketches the critical layer depth as function of wind speed for three estimates of the critical values of $\tilde{d}$ and thus complements table (2).

\section{Sensitivity of transient Ekman flows to the characteristics of the upper ocean}

In the previous section we analyzed the sensitivity of Ekman flows to the presence of stratification, the strength of stratification in the pycnocline and the depth of the mixed layer confining our consideration to the steady state situation. Here, we consider the generic case when the wind and the Ekman response are varying with time. Since the problem is linear, we, without loss of generality, characterize the wind and the Ekman response by their Fourier amplitude spectra. As in the previous section, here we will focus on the sensitivity of surface manifestations of transient Ekman flows to the same basic characteristics of the upper ocean stratification (the strength of stratification in the pycnocline and the depth of the mixed layer). For each Fourier harmonic we compare the model predictions for two limiting cases: (a) the case of infinitely strong stratification in the second layer (Elipot \& Gille model [7]), and, (b) the case of no stratification (Ekman model [6]). By choosing threshold $\delta_{\{E G-C E\}}$ which characterizes the discrepancy between the models, we can estimate the depth of the mixed layer where for a chosen frequency we can detect the presence of stratification in the surface velocity field at this frequency. We provide a table (3) which illustrates how the critical depth for various chosen $\delta_{\{E G-C E\}}$,

$$
d_{\delta_{\{E G-C E\}}} \approx \frac{-1}{\sqrt{2}}\left(\left|\frac{f+\omega}{\nu_{e 1}}\right|\right)^{-1 / 2} \ln \left(\frac{\delta_{\{E G-C E\}}}{2}\right),
$$

depends on the mixed layer eddy viscosity and chosen frequency.

A similar comparison of the two-layer model solutions with the predictions of the classical one-layer Ekman model leads to very similar conclusions, therefore, we skip their discussion. Instead, in figure 8 we provide a sample plot illustrating the Ekman response to a sharp increase of wind from 0 to $10 \mathrm{~m} \mathrm{~s}^{-1}$ in all three models (two-layer, one-layer Ekman and Elipot \& Gille).

In accordance with our previous analysis for a shallow mixed layer the surface current exhibits noticeable discrepancies between the predictions of the three models, the discrepancies decay with increase of the mixed layer thickness. A comprehensive analysis of the parameter dependence goes beyond the scope of this work. The main new point this figure aims to illustrate is that the inertial oscillations, which are the salient feature of the Ekman response, prove to be the most sensitive to the presence of stratification. In particular, the oscillations live longer than in the Ekman model.

To complement our analytical analysis based on the transfer function and provide a different viewpoint consider a few visual examples of the evolution of surface current for a variety of parameters. Figures 9 and 10 based upon the two-layer model illustrate the sensitivity of the Ekman transient current caused by a sharp increase of wind (from zero to $10 \mathrm{~ms}^{-1}$ ) to two parameters: the value of eddy viscosity in and the mixed layer thickness. 


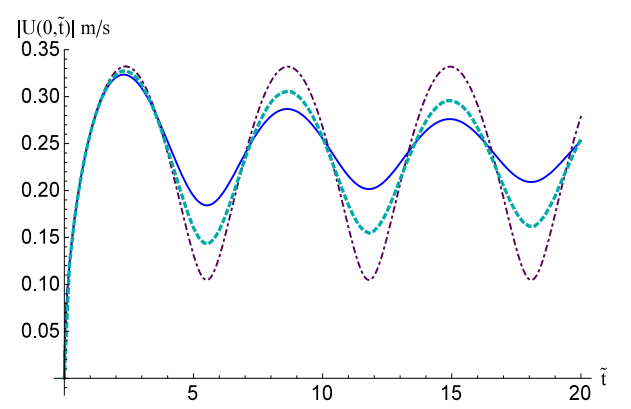

(a)

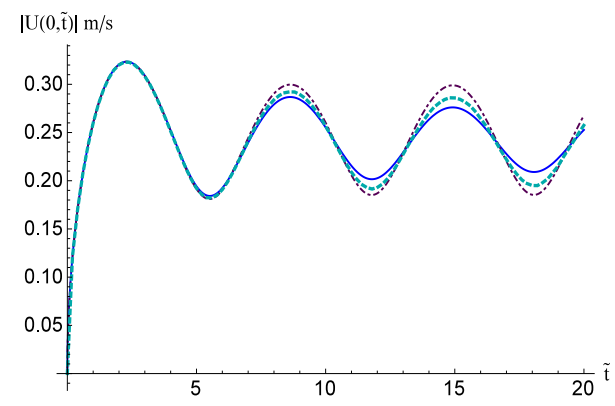

(c)

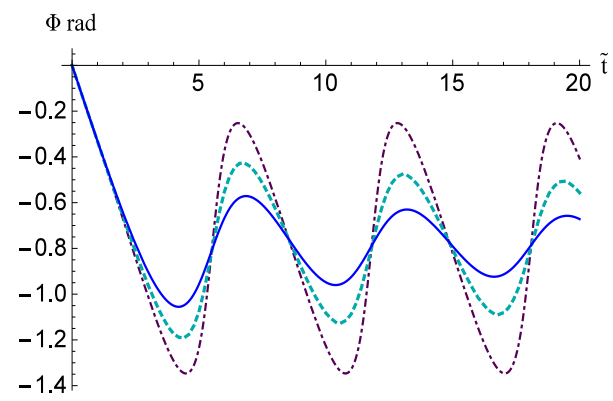

(b)

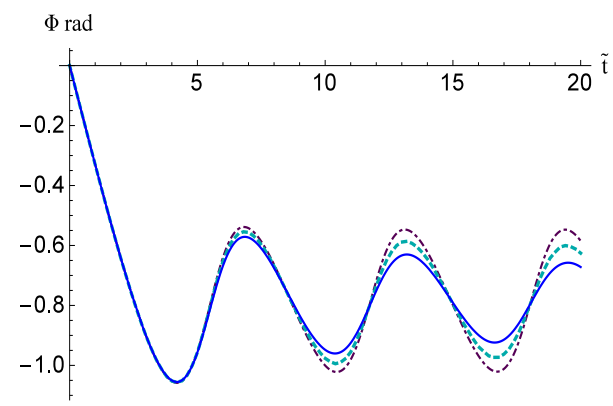

(d)

Figure 8: Surface current caused by a rapid increase of wind from 0 to $10 \mathrm{~m} \mathrm{~s}^{-1}$ in different models: the classical Ekman model (solid line), the Elipot \& Gille one-layer model with vanishing shear stress at the bottom of the mixed layer (dot-dashed line), and the two-layer model (dashed line). (a,b): $d=15 \mathrm{~m} ;(\mathrm{c}, \mathrm{d}): d=30 \mathrm{~m}$. Other parameters: $f=10^{-4} \mathrm{~s}^{-1}, \rho=1027 \mathrm{~kg} \mathrm{~m}^{-3}$, $\tau_{0}=0.175 \mathrm{~N} \mathrm{~m}^{-2}, \nu_{e 1}=5 \times 10^{-3} \mathrm{~m}^{2} \mathrm{~s}^{-1}$ and $\nu_{e 2}=5 \times 10^{-4} \mathrm{~m}^{2} \mathrm{~s}^{-1}$.

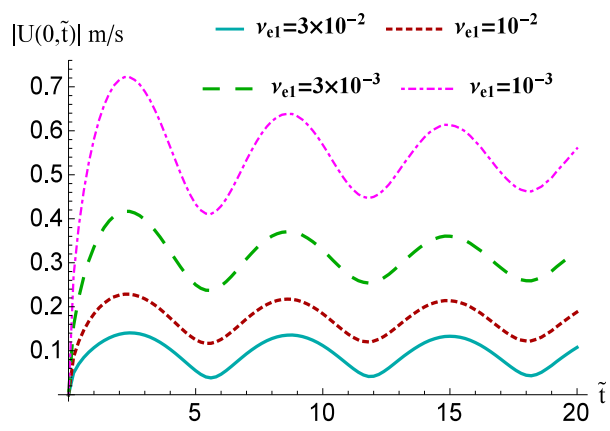

(a)

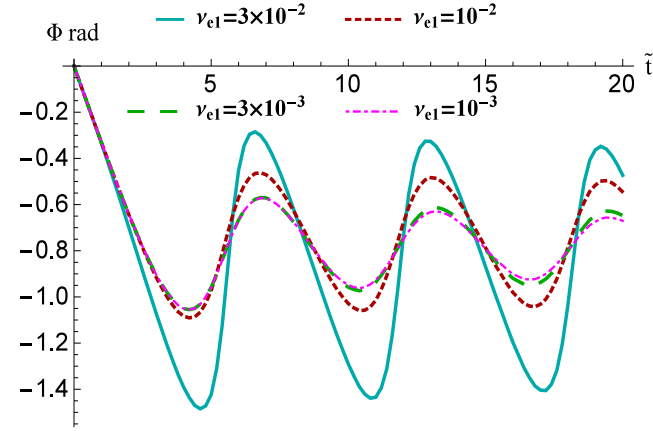

(b)

Figure 9: Surface current caused by a sharp increase of wind from 0 to $10 \mathrm{~m} \mathrm{~s}^{-1}$ in two-layer model with different eddy viscosities $\left(\nu_{e 1}\right)$ in the upper layer. Shallow mixed layer: $d=30 \mathrm{~m}$. (a) The magnitude of the surface current. (b) The deflection angle of the surface current to the wind direction. The parameter values: $f=10^{-1} \mathrm{~s}^{-1}, \rho=1027 \mathrm{~kg} \mathrm{~m}^{-3}, \tau_{0}=0.175 \mathrm{~N} \mathrm{~m}^{-2}$, $\nu_{e 2}=3 \times 10^{-4} \mathrm{~m}^{2} \mathrm{~s}^{-1}$. 


\begin{tabular}{|c|c|c|c|c|c|}
\hline$\omega \operatorname{rads}^{-1}$ & $\nu_{e} \mathrm{~m}^{2} \mathrm{~s}^{-1}$ & $d_{0.1} \mathrm{~m}$ & $d_{0.2} \mathrm{~m}$ & $d_{0.3} \mathrm{~m}$ & $d_{0.5} \mathrm{~m}$ \\
\hline $2 \pi / 1 \mathrm{hr}$ & $\begin{array}{c}2.4 \times 10^{-3} \\
5 \times 10^{-3} \\
7 \times 10^{-3} \\
10^{-2} \\
10^{-1} \\
1\end{array}$ & $\begin{array}{c}2.4 \\
3.5 \\
4.1 \\
4.9 \\
15.6 \\
49.3\end{array}$ & $\begin{array}{c}1.9 \\
2.7 \\
3.2 \\
3.8 \\
12 \\
37.9\end{array}$ & $\begin{array}{c}1.5 \\
2.2 \\
2.6 \\
3.1 \\
10 \\
31.2\end{array}$ & $\begin{array}{c}1.1 \\
1.6 \\
1.9 \\
2.3 \\
7.2 \\
22.8\end{array}$ \\
\hline $2 \pi / 10 \mathrm{hr}$ & $\begin{array}{c}2.4 \times 10^{-3} \\
5 \times 10^{-3} \\
7 \times 10^{-3} \\
10^{-2} \\
10^{-1} \\
1\end{array}$ & $\begin{array}{c}6.2 \\
9 \\
10.7 \\
12.8 \\
40.4 \\
127.8 \\
\end{array}$ & $\begin{array}{c}4.8 \\
6.9 \\
8.2 \\
9.8 \\
31 \\
98.3 \\
\end{array}$ & $\begin{array}{c}4 \\
5.7 \\
6.8 \\
8 \\
25.6 \\
81 \\
\end{array}$ & $\begin{array}{c}3 \\
4.2 \\
4.9 \\
5.9 \\
18.7 \\
59.2 \\
\end{array}$ \\
\hline$\omega \ll f$ & $\begin{array}{c}2.4 \times 10^{-3} \\
5 \times 10^{-3} \\
7 \times 10^{-3} \\
10^{-2} \\
10^{-1} \\
1\end{array}$ & $\begin{array}{c}10.4 \\
15 \\
17.7 \\
21.2 \\
67 \\
211.8\end{array}$ & $\begin{array}{c}8 \\
11.5 \\
13.6 \\
16.3 \\
51.5 \\
162.8\end{array}$ & $\begin{array}{c}6.6 \\
9.5 \\
11.2 \\
13.4 \\
42.4 \\
134.1\end{array}$ & $\begin{array}{l}4.8 \\
6.9 \\
8.2 \\
9.8 \\
31 \\
98\end{array}$ \\
\hline
\end{tabular}

Table 3: Critical depth of the mixed layer for different frequencies and threshold discrepancies $\delta_{\{E G-C E\}}$ between the predictions of the Elipot\& Gille model and the classical Ekman model for the density uniform fluid. The table shows the critical depth $d_{\delta_{\{E G-C E\}}}$ in meters for the chosen relative discrepancy $\delta_{\{E G-C E\}}$ between the two model predictions for surface velocity, the values of the chosen threshold relative discrepancy $\delta_{\{E G-C E\}}$ are indicated by the subscript.

The main conclusions we can make are simple and robust:

(i) An order of magnitude increase of the eddy viscosity $\nu_{1}$ leads to a more than twofold decrease of the mean Ekman current speed and amplitude of near-inertial oscillations, independently of the depth of the mixed layer.

(ii) The deflection of the surface current with respect to the direction of wind is sensitive to viscosity only in the case of shallow mixed layer, then, counter intuitively, inertial oscillations of the surface current direction are most pronounced for the largest viscosity; in the case of a thick mixed layer neither the mean deflection nor its inertial oscillations are sensitive to the viscosity. Our overall conclusion is the Ekman response is quite sensitive to the viscosity in the mixed layer, we note an increase of the timescale of the Ekman layer 
acceleration with the increase of viscosity. Thus, the amplitudes of near inertial oscillations (NIOs) might be considered as candidates for remote probing of bulk eddy viscosity in the mixed layer.

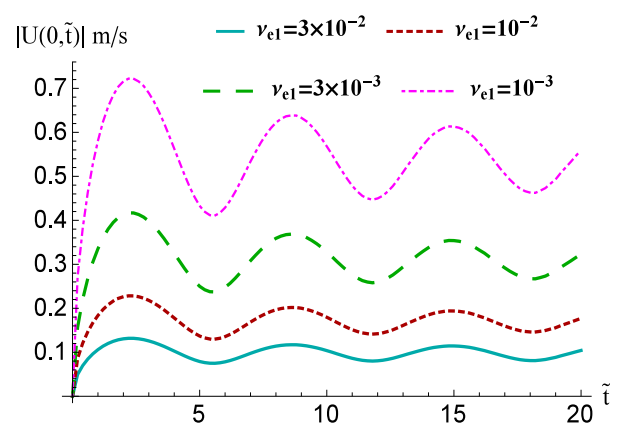

(a)

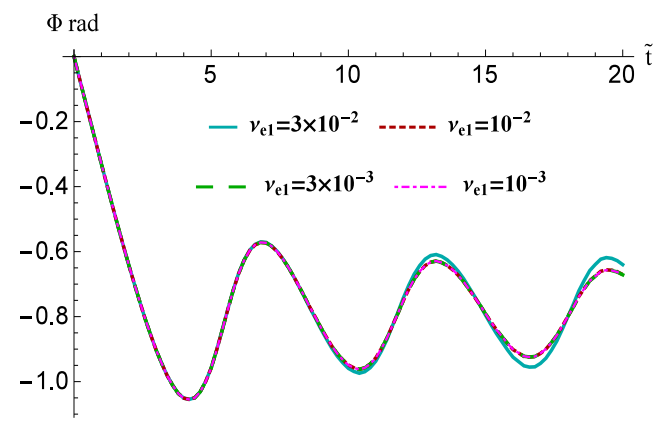

(b)

Figure 10: Surface current caused by a sharp increase of wind from 0 to $10 \mathrm{~m} \mathrm{~s}^{-1}$ in the twolayer model with different eddy viscosity values $\left(\nu_{e 1}\right)$ in the thick upper layer $(d=100 \mathrm{~m})$. (a) The magnitude of the surface current. (b) The deflection angle of the surface current to the wind direction. The parameter values: $f=10^{-1} \mathrm{~s}^{-1}, \rho=1027 \mathrm{~kg} \mathrm{~m}^{-3}, \tau_{0}=0.175 \mathrm{~N} \mathrm{~m}^{-2}$, $\nu_{e 2}=3 \times 10^{-4} \mathrm{~m}^{2} \mathrm{~s}^{-1}$.

\section{Can the upper ocean stratification profile be probed remotely by $\mathrm{HF}$ radars?}

Of great importance would have been a possibility of remote sensing the following characteristics of the upper ocean: turbulence level in the mixed layer, the presence/absence of diurnal stratification, the depth of the mixed layer and the strength of stratification in the pycnocline. In the previous section within the framework of two-layer model we examined how the speed and direction of the surface current depend on the depth of the mixed layer, eddy viscosity in the mixed layer and below. Although the surface velocity now can be measured remotely from satellites with improving accuracy and resolution, at present it is not clear when high-accuracy satellite measurements of ageostrophic currents will become available. In contrast, observations from the shore by 'High-Frequency' (HF) radars have been a widely used tool of monitoring of the sea-surface for more than thirty years. In particular, the sea-echo Doppler spectra of $\mathrm{HF}$ radars are used for probing surface currents (e.g. [1], [14], [27]), while the search continues for ways of remote sensing of other aspects of air-sea interaction (e.g. [20]). Here we briefly discuss additional possibilities provided by HF radars in view of potential remote sensing of the characteristics of the upper ocean listed above. 


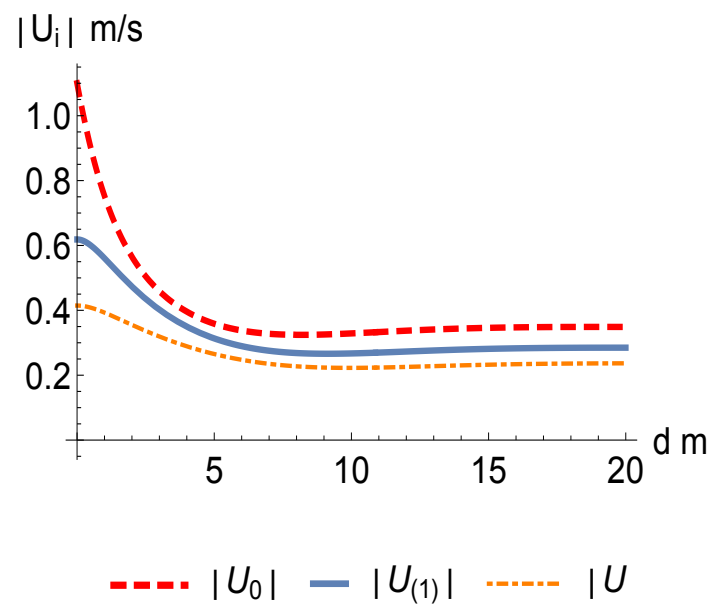

(a)

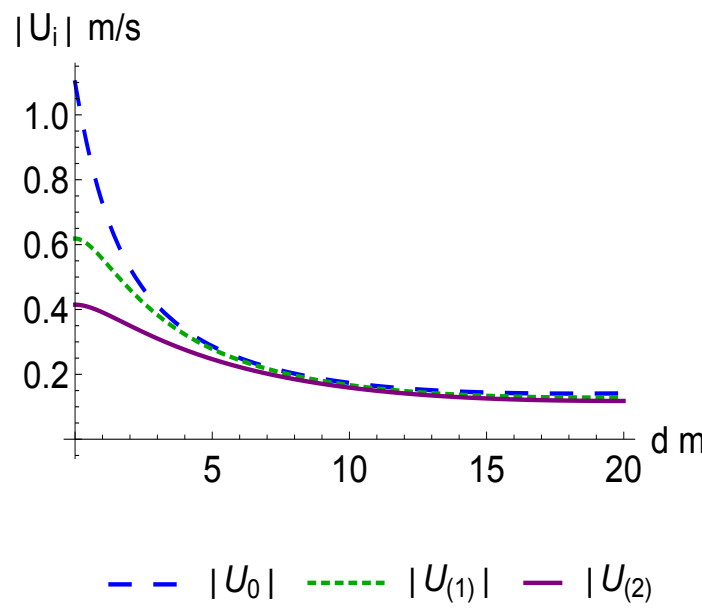

(c)

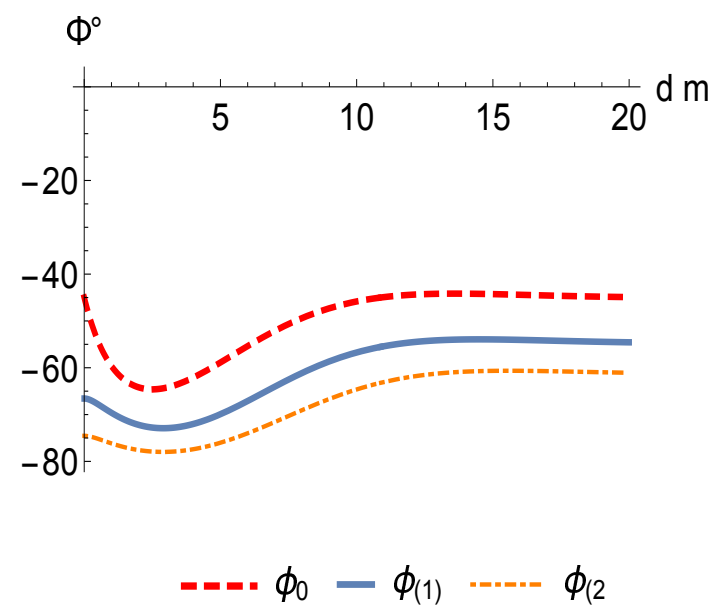

(b)

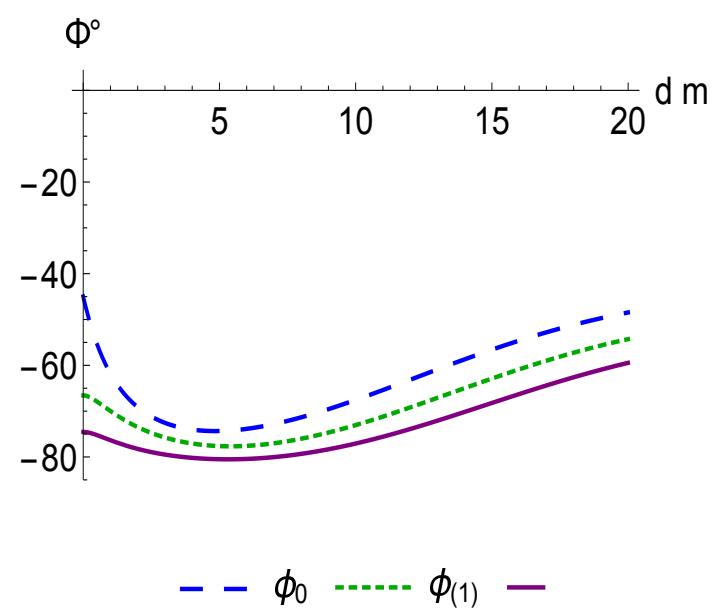

(d)

Figure 11: The velocity fields $\boldsymbol{U}_{0}$ (velocity at the surface), $\boldsymbol{U}_{(1)}$ and $\boldsymbol{U}_{(2)}$ of the steady Ekman current caused by $10 \mathrm{~m} / \mathrm{s}$ wind $\left(U_{10}=10 \mathrm{~m} / \mathrm{s}\right)$ as predicted by the 2-layer model with the eddy viscosity in the lower layer $\nu_{e 2}=2.4 \times 10^{-4} \mathrm{~m}^{2} \mathrm{~s}^{-1}$. (a,b) turbulent viscosity $\nu_{e 1}=2.4 \times 10^{-3}$ $\mathrm{m}^{2} \mathrm{~s}^{-1}$ in the upper layer; $(\mathrm{c}, \mathrm{d}), \nu_{e 1}$ is five times larger. Other parameters: $f=10^{-4} \mathrm{~s}^{-1}, \rho=$ $1027 \mathrm{~kg} \mathrm{~m}^{-3}$ and $\tau_{0}=0.175 \mathrm{~N} \mathrm{~m}^{-2}$.

The HF (high frequency) radar measurements are utilizing the fact that in the HF range of frequencies electromagnetic waves are scattered by random wavy water surface in the Bragg regime, where a monochromatic electromagnetic wave emitted with a wavenumber $k_{E}$ is reflected back primarily by the resonant Bragg wave number $k_{B}=k_{E} / 2$ of the water surface. By analyzing the Doppler spectra which exhibit easily identifiable Bragg peaks it is straightforward to find the phase velocity of the wave components associated with the peaks. By subtracting the phase velocities prescribed by the linear dispersion relation one gets a correction due to the surface currents. Although the main contribution to the EM scattering comes from the Bragg resonant wave of length $2 \pi / k_{B}$ (the main peaks), water waves are weakly nonlinear and each free Fourier 


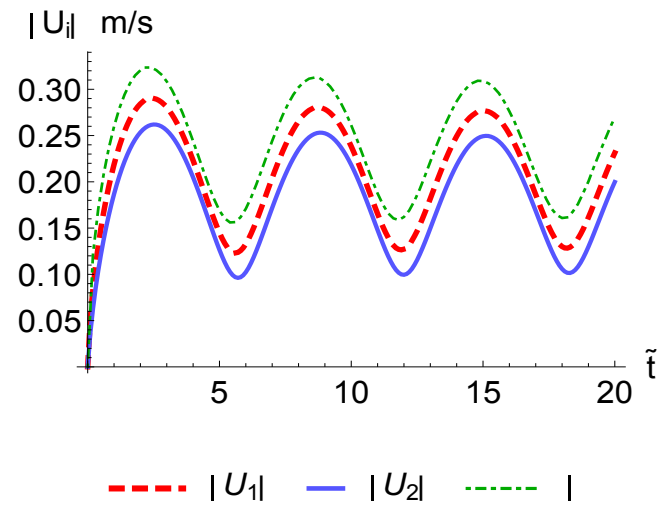

(a)

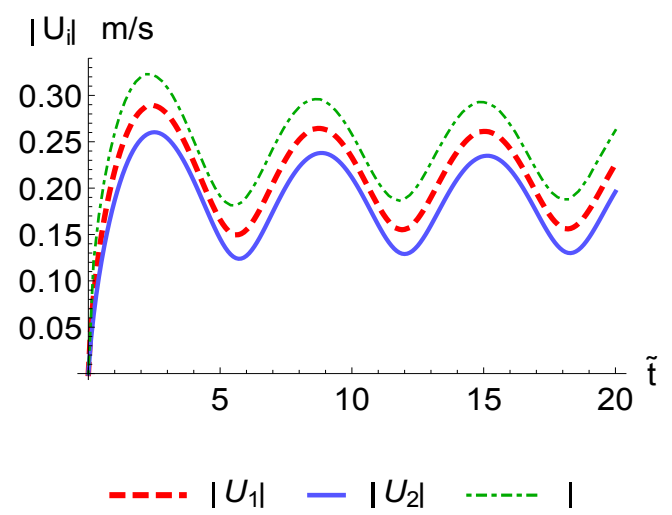

(c)

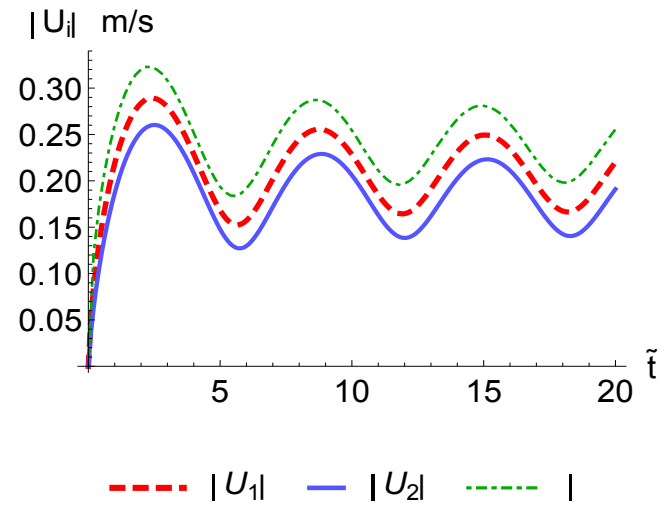

(e)

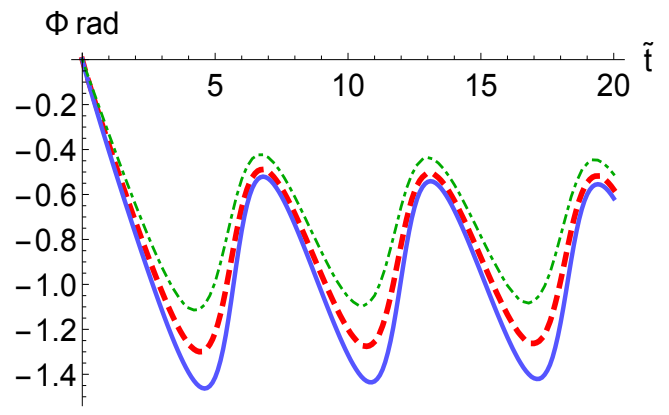

-n- $\Phi_{1}-\Phi_{2}$

(b)

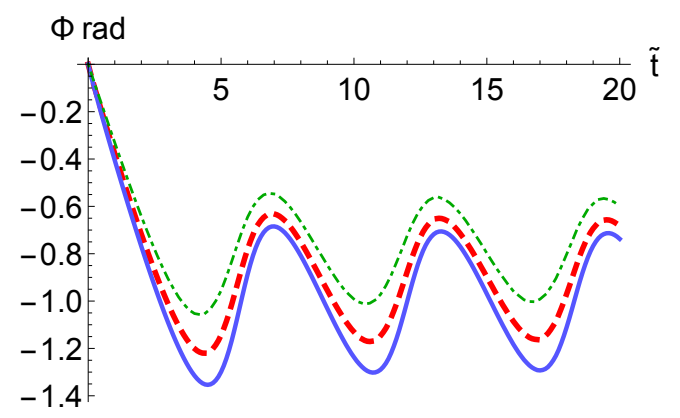

$=-\Phi_{1}-\Phi_{2}$

(d)

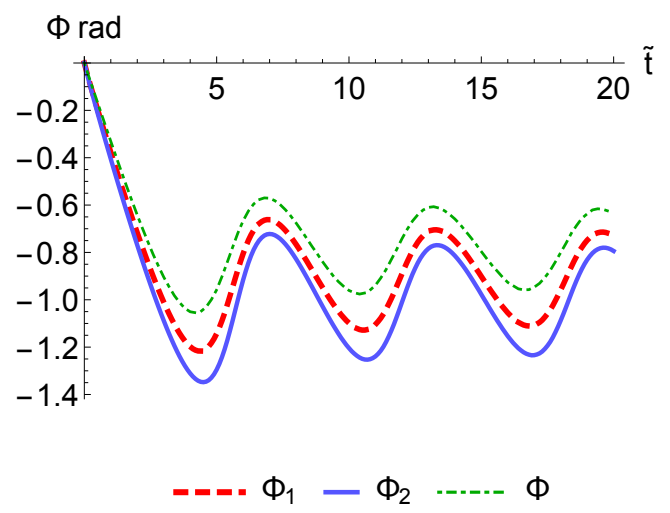

(f)

Figure 12: Transient Ekman currents caused by a sharp increase of wind from 0 to $10 \mathrm{~m} \mathrm{~s}^{-1}$ as seen by $\mathrm{HF}$ radar for various characteristics of mixed layer and stratification. Notation: $\boldsymbol{U}_{0} \equiv \boldsymbol{U}(0)$ (dot-dashed line), $\boldsymbol{U}_{(1)}$ (dashed line), $\boldsymbol{U}_{(2)}$ (solid line). (a,b): $d=20 \mathrm{~m} ;$ (c,d): $d=30 \mathrm{~m} ;(\mathrm{e}, \mathrm{f}): d=40 \mathrm{~m}$. Other parameters: $f=10^{-4} \mathrm{~s}^{-1}, \rho=1027 \mathrm{~kg} \mathrm{~m}^{-3}, \tau_{0}=0.175 \mathrm{~N} \mathrm{~m}^{-2}$, $\nu_{e 1}=5 \times 10^{-3} \mathrm{~m}^{2} \mathrm{~s}^{-1}$ and $\nu_{e 2}=5 \times 10^{-5} \mathrm{~m}^{2} \mathrm{~s}^{-1}$.

component also has bound waves. The second harmonic of the twice longer water wave has the 
same resonant wavenumber $k_{B}$. These bound waves associated with a free wave of wavelength twice the Bragg one are responsible for the second harmonic peak in the radar spectra ([21]). The correction due to surface current depends on the wavenumber under consideration. With a good accuracy it could be approximated by the Stewart-Joy formula ([25]). Applying the Stewart-Joy formula to the wave corresponding to the main Bragg peak and to the second contributor to the scattering - the wave of twice the the Bragg wavelength one gets two integrals of the current,

$$
\boldsymbol{U}_{(1)}=2\left|\boldsymbol{k}_{B}\right| \int_{0}^{\infty} \boldsymbol{U}(z, t) e^{-2\left|\boldsymbol{k}_{B}\right| z} d z, \quad \boldsymbol{U}_{(2)}=\left|\boldsymbol{k}_{B}\right| \int_{0}^{\infty} \boldsymbol{U}(z, t) e^{-\left|\boldsymbol{k}_{B}\right| z} d z
$$

where $\boldsymbol{U}(z, t)$ is the velocity profile and $\left|\boldsymbol{k}_{B}\right|=2 \pi / \lambda$ is the Bragg wavenumber. For the sake of certainty in our further consideration we have chosen a commonly used radar frequency $12 \mathrm{MHz}$ which corresponds to the water wave wavelength $\lambda=18 \mathrm{~m}$. In the previous section we examined how the properties of the upper ocean manifest themselves in the field of surface velocity $\boldsymbol{U}_{0}$. The surface velocity is routinely measured by HF radars and $\boldsymbol{U}_{(1)}$ is usually taken as $\boldsymbol{U}_{0}$. Here, in figures 11 and12 we show how sensitive is the discrepancy between the two integrated velocities $\boldsymbol{U}_{(1)}$ and $\boldsymbol{U}_{(2)}$ and the true surface velocity $\boldsymbol{U}_{0}$ to the depth of the mixed layer and to the values of eddy viscosity in each layer. The figure also shows how the discrepancies in the current deflection angle depend on the depth of the mixed layer and to the values of eddy viscosity. The figure suggests that the discrepancies are quite substantial for the diurnal stratification, which looks very promising for its remote probing. Moreover, the discrepancies in the deflection angle remain discernible for the seasonal stratification. A more thorough study is needed to explore the potential of these findings for remote sensing of the upper ocean.

An overview of the collection of examples (figures 11,12) presented in this section enables us to conclude that there are measurable discrepancies between the true surface velocity $\boldsymbol{U}_{0}$ and the surface velocities $\boldsymbol{U}_{(1)}, \boldsymbol{U}_{(2)}$ retrieved by HF radars for a wide range of oceanic conditions. These discrepancies can provide quantitative estimates of the level of turbulence in the mixed layer and the thickness of of the mixed layer. To a lesser extent, these discrepancies can constrain the estimates of the stratification in the pycnocline. Once applied to real field observations the above results can provide a valuable insight into an important aspect of air-sea interaction.

\section{Concluding remarks}

As a first step towards a theoretical framework for remote sensing of upper ocean stratification we explore the idea that the stratification affects dynamics of the surface Ekman current, crucially, we assume that we can measure the surface current with a good accuracy. To link the stratification and surface current in this work we introduced and examined a novel two-layer model of the Ekman boundary layer which utilizes the great disparity in magnitudes of eddy viscosity in the mixed layer and in the stratified flow below. Assuming the time dependence of horizontally uniform wind stress $\boldsymbol{\tau}(t)$ on the surface to be given we found the boundary layer response to such varying surface stress $\boldsymbol{\tau}(t)$ in a closed analytical form. Thus, for an arbitrary $\boldsymbol{\tau}(t)$ we found and 
analyzed the time-dependent solution which describes dynamics of the Ekman boundary layer in such a model, which is a novel exact solution to the full Navier-Stokes equations. We examined various steady and unsteady regimes within the framework of the adopted model. It has been verified that in the corresponding limits the solutions tend to the classical Ekman solution for infinitely deep fluid or to the Elipot \& Gille solution one in the limit of vanishing eddy viscosity in the stratified layer. The model is of interest per se, apart from the remote sensing context.

The solution reveals the limitations of the model. Under stronger winds the solution exhibits a strong shear at the bottom of the mixed layer, which can cause instability of the interface. This might be an important physical mechanism of the mixed layer deepening and entrainment. To quantify this entrainment mechanism one has to adopt one of the existing parameterizations of the eddy viscosity in the stratified fluid, which goes beyond the scope of this study. In the regime of the mixed layer deepening a more appropriate boundary condition at the bottom of the mixed layer would be the constancy of the Richardson number, $R i=1 / 4$, as was suggested by ([11]). The account for mixing at the bottom of the mixed layer needs a dedicated study.

From the perspective of remote sensing of the basic characteristics of the mixed layer (such as its thickness, the eddy viscosity) and stratification below, we analyzed the sensitivity of the surface manifestations of the Ekman currents provided by the time-dependent solution for the surface current $\boldsymbol{U}_{0}(t)$ for various regimes. We found that for a wide range of parameters and regimes of evolution the presence of stratification does manifest itself in the field of the surface current $\boldsymbol{U}_{0}(t)$. The manifestations on the surface have proved to be most sensitive to the non-dimensional depth of the mixed layer $\tilde{d}=d / \delta_{1}, \delta_{1}=\sqrt{2 \nu_{e 1} / f}$ : the manifestations on are discernible for $\tilde{d} \leq 1.5$. For the seasonal stratification this condition is always satisfied under sufficiently strong winds. The amplitudes of near inertial oscillations (NIOs) proved to be quite sensitive to the mixed layer bulk eddy viscosity $\nu_{e 1}$, which makes NIOs a candidate worthy of consideration for remote sensing of the mixed layer turbulence. However, since the NIOs amplitudes are also very sensitive to the mixed layer thickness and the stratification below, it makes the task of directly evaluating the turbulence level very challenging, if not impossible.

Thus, different elements of the Ekman response are sensitive to different characteristics of the upper ocean. A proper theory of NIOs generation is lacking, a dedicated effort is needed to explore further the NIO's potential for remote sensing.

The potential of advancing in remote probing of the upper ocean stratification profile by using HF radars has been also examined. By utilizing both the main and second harmonic peaks of the HF radar echo, which enables one to probe the boundary layer at two different effective depths, one obtains new constraints on the mixed layer depth and parameters of stratification.

Although our analysis of the possibilities of retrieving the upper ocean stratification and eddy viscosity characteristics from the surface velocity field is based on the exact solutions of the Navier-Stokes equation, the adopted hydrodynamic model implicitly relies on a number of key assumptions whose role is not obvious at the moment. In particular, as we mentioned, we've ignored mixing and entrainment at the bottom of the mixed layer. Moreover, currently, we do not know how good is the Ekman paradigm in capturing reality, we are unaware of sufficiently detailed verification studies and have recently articulated our doubts in [19]). How crucial are these uncertainties for the broad picture remains to be investigated. It is also known 
that the account of wave induced Stokes drift might result in order one contribution to the flow velocity at the surface which we take as one of the key inputs into our reconstruction of the stratification profile. The account of the Stokes drift is relatively straightforward, but it is very detrimental for the simplicity of the model (e.g. [23]). Overall, at present we do not know what addressing these issues might bring, but expect it will not change the picture qualitatively. We expect our main qualitative conclusion to remain robust, that is, the presence of diurnal and seasonal stratification noticeably alters the ageostrophic part of the surface velocity field when the thickness of the mixed layer $d$ is about the Ekman scale $\delta_{1},\left(\delta_{1}=\sqrt{2 \nu_{e 1} / f}\right)$ or less. Our overall conclusion is that the outlined route of remote probing of the upper ocean stratification is feasible in the foreseeable future, but needs dedicated efforts both at the theoretical/modelling side and at the experimental/technical side. The techniques of retrieving the surface velocity field (apart from HF radars) have to be significantly improved.

\section{References}

[1] Broche, P., Forget, P., De Maistre, J.C., Devenon, J.L. And Crochet, M., 1987. VHF radar for ocean surface current and sea state remote sensing. Radio Science, $22(1)$, pp.69-75.

[2] Chapron, B., Collard, F., Ardhuin, F., 2005. Direct measurements of ocean surface velocity from space: Interpretation and validation. J. Geophys. Res. 110, C07008. http://dx.doi.org/10.1029/2004JC002809

[3] Chelton, D.B., Schlax, M.G., Samelson, R.M., Farrar, J.T., Molemaker, M.J., MCWilliams, J.C. AND GulA, J., 2019. Prospects for future satellite estimation of smallscale variability of ocean surface velocity and vorticity. Progress in Oceanography, 173, pp.256350.

[4] Coleman, G.N., Ferziger, J.H. and Spalart, P.R., 1990. A numerical study of the turbulent Ekman layer. Journal of Fluid Mechanics, 213, pp.313-348.

[5] D'Asaro, E.A. And Dairiki, G.T., 1997. Turbulence intensity measurements in a winddriven mixed layer. Journal of Physical Oceanogr,, 27(9), pp.2009-2022.

[6] Ekman, V. W. 1905. On the influence of the Earth's rotation on ocean currents. Arch. Math.Astron. Phys.2, 1-52.

[7] Elipot, S. \& Gille, S. T. 2009. Ekman layers in the Southern ocean: spectral models and observations, vertical viscosity and boundary layer depth. Ocean Sci., 5, 115-139.

[8] Emanuel, K.A., 1988. The maximum intensity of hurricanes. Journal of the Atmospheric Sciences, 45(7), pp.1143-1155.

[9] Huang, N.E., 1979. On surface drift currents in the ocean. Journal of Fluid Mechanics, 91(01), pp.191-208. 
[10] Kudryavtsev, V.N. And A.V.Soloviev, 1990. Slippery nearsurface layer of the ocean arising due to daytime solar heating, J. Phys. Oceanogr., 20, 61717628, doi:10.1175/ 15200485(1990)020

[11] Kudryavtsev, V., Monzikova, A., Combot, C., Chapron, B. and Reul, N., 2019. A simplified model for the baroclinic and barotropic ocean response to moving tropical cyclones: 2. Model and Simulations. Journal of Geophysical Research: Oceans, 124(5), pp.3462-3485.

[12] Lewis, D. M. \& Belcher, S. E. 2004. Time-dependent, coupled, Ekman boundary layer solutions incorporating Stokes drift, Dynam. Atmos. Oceans, 37, 313-351.

[13] McWilliams, J.C., Sullivan, P.P. And Moeng, C.H., 1997. Langmuir turbulence in the ocean. Journal of Fluid Mechanics, 334, pp.1-30.

[14] Paduan, J.D. And Graber, H.C., 1997. Introduction to high-frequency radar: reality and myth. Oceanography, 10(2), pp.36-39.

[15] Phillips, O. M. 1977. The dynamics of the upper ocean, Cambridge University Press.

[16] Price, J. F., \& Sundermeyer, M. A. 1999. Stratified Ekman layers. Journal of Geophysical Research: Oceans, 104(C9), 20467-20494.

[17] Price, J.F., Weller, R.A. And Pinkel, R., 1986. Diurnal cycling: Observations and models of the upper ocean response to diurnal heating, cooling, and wind mixing. Journal of Geophysical Research: Oceans, 91(C7), 8411-8427.

[18] Schade, L.R. And Emanuel, K.A., 1999. The ocean's effect on the intensity of tropical cyclones: Results from a simple coupled atmosphere?ocean model. Journal of the Atmospheric Sciences, 56(4), pp.642-651.

[19] Shrira, V. I., And Almelah, R.B. 2020. Upper-ocean Ekman current dynamics: a new perspective, J. Fluid Mech., 887, A24-1-A24-32; doi: http://dx.doi.org/10.1175/JPO-D-140247.1

[20] Shrira, V. I., And Forget, P. 2017. On the Nature of Near-Inertial Oscillations in the Uppermost Part of the Ocean and a Possible Route towards HF Radar Probing of Stratification, J. Phys. Oceanogr., 45, 2660-2678;

doi: http://dx.doi.org/10.1175/JPO-D-14-0247.1

[21] Shrira, V.I., Ivonin, D.V., Broche, P. And de Maistre, J.C., 2001. On remote sensing of vertical shear of ocean surface currents by means of a Singlefrequency VHF radar. Geophysical research letters, 28(20), pp.3955-3958.

[22] Soloviev, A. And Lucas, R. 2006. The Near-Surface Layer of the Ocean, Springer, 552 pp. 
[23] Sullivan, P.P. and MCWilliams, J.C., 2010. Dynamics of winds and currents coupled to surface waves. Annual Review of Fluid Mechanics, 42, 19-42.

[24] Sutyrin, G. G., And A. P. Khain, 1984. Effect of the ocean-atmosphere interaction on the intensity of a moving tropical cyclone. Atmos. Ocean. Phys., 20, 697703.

[25] Stewart, R.H. And Joy, J.W., 1974, HF radio measurements of surface currents. Deep-sea Res. 21, (12), 1039-1049.

[26] Weller, R.A. And Price, J.F., 1988. Langmuir circulation within the oceanic mixed layer. Deep Sea Research Part A. Oceanographic Research Papers, 35(5), pp.711-747.

[27] Wyatт, L.R., 2000. Limits to the inversion of HF radar backscatter for ocean wave measurement. Journal of Atmospheric and Oceanic Technology, 17(12), pp.1651-1666.

[28] Zervakis, V., Kokkini, Z. And Potiris, E., 2017. Estimating Mixed Layer Depth with the use of a coastal High-Frequency radar. Continental Shelf Research, 149, pp.4-16. 